\title{
Flora de la costa de OAXaca, MÉXICO: Lista florística de la REGIÓN DE ZIMATÁN
}

\author{
Silvia H. Salas-Morales, Alfredo Saynes-Vásquez y Leo Schibl \\ Sociedad para el Estudio de los Recursos Bióticos de Oaxaca (SERBO, A.C.). Porfirio Díaz No. 211, Centro. \\ Oaxaca, Oax. C.P. 68000. Teléfono: (951) 516-00-98. Correo electrónico: serbo@prodigy.net.mx
}

\begin{abstract}
Resumen: La región de Zimatán, Oaxaca, cuenta con varios tipos de vegetación y una gran diversidad florística, debido principalmente a un amplio intervalo altitudinal que varía desde el nivel del mar hasta 2,580 m. En 1991 está región fue propuesta como un área de gran importancia para la conservación en el estado de Oaxaca, sobre todo por las amplias superficies de selvas tropicales secas en excelente estado de conservación; sin embargo, la escasa información biológica disponible condujo a realizar a partir de 1997 una serie de estudios que sustentaran su conservación. En este trabajo se presenta la información florística de 8,063 colectas originales realizadas desde entonces. La lista florística incluye 1,384 especies, 668 géneros y 144 familias, lo que hace que la región de Zimatán sea una de las mejor colectadas y con mayor diversidad florística en la costa mexicana del Pacífico, junto con la Estación Biológica de Chamela en Jalisco.
\end{abstract}

Palabras clave: Flora, vegetación, Costa del Pacífico, Oaxaca, México.

\begin{abstract}
The Zimatan region of Oaxaca is characterized by the occurrence of several vegetation types and a large floristic diversity, primarily due to the large elevational range that it encompasses (from sea level to 2,580 m). In 1991 this region was proposed as a high-priority area for conservation in Oaxaca State, largely due to its extensive, well-preserved tracts of tropical dry forest. Nonetheless, little biological information is available for this area. In 1997 we initiated a series of studies aimed at supporting the conservation of the Zimatan region. Here we present floristic data based on 8,063 original botanical collections. The list includes 1,384 vascular plant species, distributed among 668 genera and 144 families. These collections make of the Zimatan region one of the most comprehensively collected areas and most diverse on the Mexican Pacific coast, along with the Chamela Biological Station in Jalisco State.
\end{abstract}

Keywords: Flora, vegetation, Pacific Coast, Oaxaca, Mexico.

D esde el punto de vista biológico, Oaxaca está considerado como uno de los estados más diversos en el país. Su complejidad orográfica, la influencia de dos oceános y su historia geológica han contribuido de manera conjunta para dar como resultado esta vasta diversidad. En la entidad se encuentran casi todos los tipos de vegetación que Rzedowski (1978) reconoce para el país, aunque con características propias tanto de las especies que los componen como de su fisonomía. Existen diversas estimaciones acerca de la riqueza florística de Oaxaca, las cuales varían de 8,000 (Toledo, 1988) a 9,000 especies (Lorence y García-Mendoza, 1989; Rzedowski, 1991). A más de 10 años de publicadas estas cifras, aún no se ha avanzado con el inventario de la flora del estado y su magnitud todavía se mantiene en estimaciones.

García-Mendoza y Torres-Colín (1999) consideran que de las diez provincias fisiográfico-florísticas en las que dividen el estado, cuatro están de medianamente a bien colectadas. Entre éstas se encuentra la del Istmo de Tehuantepec, que cuenta con valiosas contribuciones a su flora (Zizumbo-Villarreal y Colunga, 1980; Torres-Colín, 1989; Torres-Colín et al., 1997; Pérez-García et al., 2001). Las otras seis regiones están consideradas como insuficientemente colectadas y algunas prácticamente son desconocidas, entre las que se incluyen a la Sierra Madre del Sur y la Planicie Costera del Pacífico, en donde se ubica la región de Zimatán. Esta región abarca un amplio intervalo altitudinal, que va desde el nivel del mar hasta casi 2,600 m, lo que se traduce en una gran diversidad de tipos de vegetación, desde selvas bajas espinosas caducifolias hasta bosques mesófilos de montaña y bosques templados de pinoencino, pasando por grandes superficies de selvas secas, subhúmedas y húmedas. Para estos tipos de vegetación se carecía de información biológica, excepto por colectas 
históricas aisladas de flora que señalaban la importancia de áreas aledañas, p.ej. Cerro Espino y alrededores de Salina Cruz (M. Sousa, com. pers.). El inventario florístico de la región de Zimatán surgió ante la necesidad de generar información que permitiera fundamentar la conservación de sus ecosistemas. En 1991 esta área fue propuesta como una región prioritaria para la conservación de la biodiversidad en Oaxaca (García et al., 1992). Posteriormente, la Comisión Nacional para el Conocimiento y Uso de la Biodiversidad (Arriaga et al., 2000) incluyó a las regiones Costa y Sierra Sur como regiones prioritarias a nivel nacional.

\section{Área de estudio}

La región de Zimatán se localiza en la costa oaxaqueña, entre el desarrollo turístico Bahías de Huatulco y el Puerto de Salina
Cruz. Cubre un área de 71,339 ha, la cual abarca las partes media y baja de la cuenca del río Zimatán y las partes bajas de las cuencas del río Chacalapa y parte del río Copalita (figura 1).

Los municipios en los que se encuentra son San Miguel del Puerto, del distrito de Pochutla, y San Pedro Huamelula, del distrito de Tehuantepec. La carretera federal No. 200 atraviesa la región de este a oeste, y de ella parten numerosos caminos de terracería que dan acceso a las comunidades asentadas ahí.

El área se ubica en la Provincia Morfotectónica de la Sierra Madre del Sur. Los terrenos de 0 a 200 m de elevación se asignan a la Subprovincia de la Planicie Costera del Pacífico, mientras que por arriba de la cota de $200 \mathrm{~m}$ corresponden a la subprovincia de las Tierras Altas de Oaxaca y Puebla, específicamente a la zona de las Cuestas del Pacífico (Ferrusquía-Villafranca, 1998).

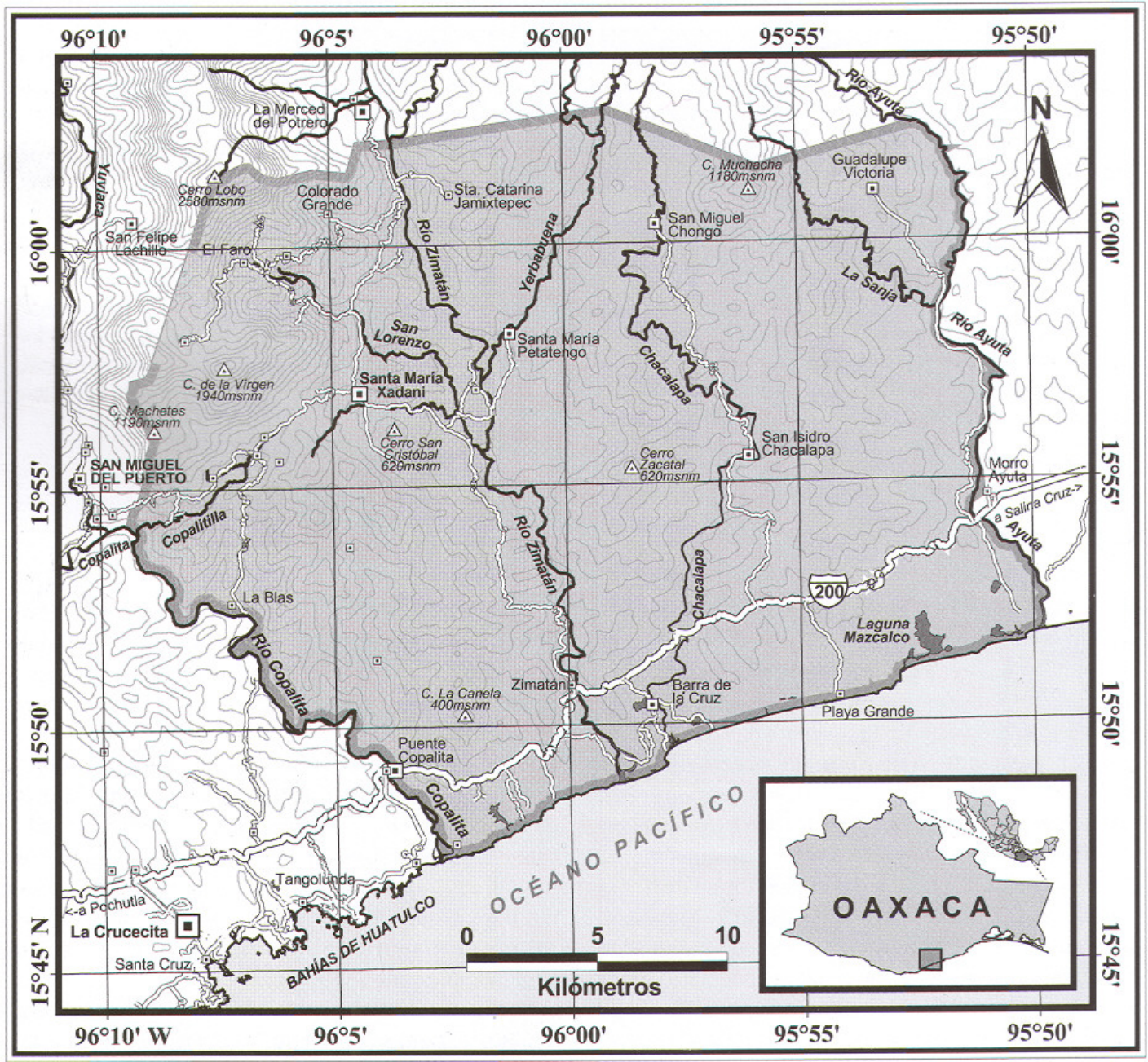

Figura 1. Ubicación del Área de Estudio 
El sustrato del área presenta una gran variedad de rocas, tanto por su origen como por su edad. Entre ellas predomina, en la porción este del área, un complejo metamórfico de edad paleozoica, y hacia la porción occidental, gneises de edad jurásica, los cuales están asociados a esquisto, granulita, granodiorita gneísica y metagranito, formando parte de la franja metamórfica del Complejo Xolapa. En manchones dispersos se encuentran afloramientos de gneises del Precámbrico, los cuales presentan una textura granoblástica y gneísica, cuyo origen es probablemente una roca granítica. La porción norte está cubierta por granito de edad jurásicacretácica. Finalmente, también en el norte, formando el sustrato del Cerro de La Virgen y del Cerro Lobo, se encuentran rocas calizas del Cretácico Inferior, las cuales son cristalinas, de facies post-arrecifal de plataforma, y están relacionadas con la Formación Teposcolula del Cretácico Inferior. En las orillas de los ríos y planicies se han depositado suelos residuales del Cuaternario y hacia la línea de costa, suelos de origen litoral (INEGI, 1984, 1988a).

De manera general, los suelos predominantes son Cambisoles crómicos, los cuales cubren la mayor parte de la región. Hacia el norte se presentan Regosoles eútricos, junto con Litosoles. En las orillas de ríos y planicies aluviales los suelos son clasificados como Feozems háplicos (INEGI, 1988b, 1989).

En la región los ríos más importantes, por llevar agua todo el año, son el Copalita, el Zimatán y el Ayuta. Todos ellos desembocan en el Océano Pacífico. En época de lluvias se convierten en corrientes torrenciales, ya que captan escurrimientos provenientes de la Sierra Madre del Sur. El Copalita es el río que recorre mayor distancia y su cuenca ha sido profundamente alterada. Debido a que el área colinda con el mar, cuenta con lagunas costeras de agua salobre entre las que destaca la de Mazcalco.

No existen estaciones meteorológicas en el área; las más cercanas se encuentran en Puerto Ángel, a $80 \mathrm{~km}$ aprox. al oeste, y en Salina Cruz, a $100 \mathrm{~km}$ aprox. al este, del área de estudio. De acuerdo a la cartografía disponible (CETENALUNAM, 1970; INEGI, 1980), en el área de estudio se presentan varios tipos climáticos, lo cual se debe a su amplio intervalo altitudinal. El clima de las partes bajas, considerando la planicie costera y los lomeríos adyacentes, es cálido subhúmedo, con lluvias en verano; su fórmula climática es Aw' ${ }_{0}(w) i g$ (según Köppen, modificado por García, 1988), el cual representa el tipo más seco de los cálidos subhúmedos. El clima que se presenta en las estribaciones de la Sierra Madre del Sur es también de tipo cálido, intermedio en cuanto al grado de humedad; su fórmula climática es $\mathrm{Aw}_{1}(\mathrm{w}) \mathrm{ig}$. Ascendiendo en la Sierra Madre del Sur, el clima continúa siendo cálido, pero es el más húmedo de los subhúmedos $\left(\mathrm{Aw}_{2}(\mathrm{w}) \mathrm{ig}\right)$. En altitudes superiores el clima es semicálido húmedo con abundantes lluvias en verano; su fórmula es $\mathrm{A}(\mathrm{C}) \mathrm{m}(\mathrm{w}) \mathrm{ig}$. Hacia las mayores altitudes de esta región, el clima se torna templado suhúmedo con lluvias en verano, cuya fórmula es $\mathrm{C}\left(\mathrm{w}^{2}\right)(\mathrm{w})$ big, el más húmedo de este grupo. En toda el área se presenta una canícula, es decir, una pequeña temporada menos lluviosa dentro de la estación de lluvias, llamada también sequía de medio verano.

\section{Métodos}

En 1997 se iniciaron los recorridos de colecta botánica de manera general en toda el región de estudio, con apoyo de los mapas temáticos y topográficos de INEGI. Posteriormente, junto con la interpretación de una imagen de satélite Landsat TM de 1999, se determinaron los tipos de vegetación presentes y su estado de conservación, así como las vías de acceso y poblados cercanos. De esta manera, se hizo un programa de recorridos de colecta intensiva. El material colectado se procesó, a partir de 1999, en el Herbario Nacional de la Universidad Nacional Autónoma de México (MEXU), en donde se contó con el apoyo de diversos especialistas. El material determinado se depositó en los herbarios GUIE' de SERBO, OAX del CIIDIR-IPN, MEXU de la UNAM y ENCB del IPN. La información obtenida en campo fue capturada en una base de datos, diseñada específicamente para SERBO en el programa ACCESS 97.

Desde el inicio del proyecto se contó con la participación de dos colectores locales, cuya permanencia en el área permitió la colecta de diversas especies en sus diferentes estadios fenológicos. Los colectores locales trabajaron con equipos de posicionamiento global, por lo que todas las colectas que se hicieron en esta región han sido correctamente ubicadas con coordenadas geográficas, lo cual permitió integrar y desplegar la información florística en mapas digitales que se tienen para el área.

La nomenclatura utilizada para los tipos de vegetación presentes en la región de estudio es la de Miranda y Hernández-X. (1963); las especies que se mencionan para cada tipo de vegetación fueron colectadas durante este trabajo. La fuente de información del listado florístico proviene también de las colectas hechas en esta investigación. El listado tiene el siguiente orden: (1) Pteridophyta, (2) Cycadophyta, (3) Coniferophyta y (4) Magnoliophyta. El arreglo de las familias de Pteridophyta se basó en Mickel y Beitel (1988), y el de las Magnoliophyta se hizo de acuerdo con el sistema de clasificación de Cronquist (1981), aunque se conserva el nombre de la familia Leguminosae por cuestiones prácticas. En cada sección las familias y especies están organizadas alfabéticamente. La nomenclatura de los géneros y las especies, así como los autores, se verificaron en la base de datos $\mathrm{W}^{3}$ TROPICOS (www.mobot.org), pero respetando las determinaciones de los diversos especialistas. Los autores se abreviaron de acuerdo con Brummit y Powell (1992). Para cada especie se presenta el número de colecta que respalda el registro, seguido por las iniciales del colector; se incluyeron sólo cuatro números de colecta por colector y por especie cuando había más que este número. En los resultados se 
presentan las especies listadas en la NOM-059-2001 (Diario Oficial de la Federación, 2002).

\section{Resultados}

Flora. La información presentada en el listado es el resultado de la determinación de 8,063 ejemplares colectados en la región de estudio dentro del proyecto Flora de las Selvas Secas de la Costa de Oaxaca, durante cuyo desarrollo se visitaron y georreferenciaron 968 sitios de colecta. El listado incluye un total de 1,384 especies y 70 infraespecies, distribuidas en 668 géneros y 144 familias (cuadro 1, apéndice 1); el total de colectas en el área de Zimatán asciende a 14,273 números.

La riqueza de géneros y especies se concentra en las siguientes familias: Leguminosae (245 especies), Asteraceae
(156), Poaceae (66), Euphorbiaceae (54), Rubiaceae (52), Boraginaceae (36), Convolvulaceae (36), Cyperaceae (35), Acanthaceae (33), Orchidaceae (33) y Malvaceae (30); en conjunto, estas 11 familias abarcan $56.1 \%$ de las especies incluidas en el listado (cuadro 2). La representación de las cuatro familias más ricas en especies es muy similar a la que se ha reportado en floras de otros sitios con tipos de vegetación similares (Lott, 1985; Reyes-García y Sousa, 1997; TorresColín et al., 1997; Peralta-Gómez et al. 2000; Pérez-García et al., 2001). Esta representación a nivel de familia coincide con el patrón que reporta Rzedowski (1991) para la flora de México.

Los géneros con el mayor número de especies son Ipomoea (22), Solanum (18), Cyperus (18), Senna (16), Cordia (16), Acacia (14), Lonchocarpus (14), Bursera (13), Eupatorium (13), Desmodium (13), Euphorbia (12), Mimosa

Cuadro 1. Datos sintéticos de la riqueza florística de la región de Zimatán.

\begin{tabular}{lcccc}
\hline Grupo & Familias & Géneros & Especies & Infraespecies \\
\hline Pteridophyta & 9 & 23 & 49 & - \\
Coniferophyta & 1 & 1 & 1 & - \\
Cycadophyta & 1 & 1 & 1 & - \\
Magnoliophyta & 133 & 643 & 1,333 & 72 \\
Total & 144 & 668 & 1,384 & 72 \\
\hline
\end{tabular}

Cuadro 2. Familias con mayor número de especies de la región de Zimatán.

\begin{tabular}{lccc}
\hline Familia & Número de géneros & Número de especies & $\begin{array}{c}\text { Proporción del total } \\
\text { de especies }(\%)\end{array}$ \\
\hline Leguminosae & 73 & 245 & 17.7 \\
Asteraceae & 74 & 156 & 11.2 \\
Poaceae & 34 & 66 & 4.8 \\
Euphorbiaceae & 20 & 54 & 4.0 \\
Rubiaceae & 27 & 52 & 3.8 \\
Convolvulaceae & 7 & 36 & 2.6 \\
Boraginaceae & 5 & 36 & 2.6 \\
Cyperaceae & 8 & 35 & 2.5 \\
Acanthaceae & 15 & 33 & 2.4 \\
Orchidaceae & 24 & 33 & 2.4 \\
Malvaceae & 15 & 30 & 2.1 \\
Total & 302 & 776 & 56.1 \\
\hline
\end{tabular}


(12), Ficus (12), Indigofera (11), Croton (11) y Heliotropium (10). Se encontraron 18 especies nuevas para la ciencia, dos de las cuales ya han sido descritas y publicadas, mientras que el resto está en proceso de descripción. Cinco registros fueron nuevos para el estado de Oaxaca; de éstos, tres son nuevos para el país (Gyrocarpus americanus, Lophostachys guatemalensis var. nov. y Aristolochia anguicida, ésta última reportada recientemente también para Nizanda (Pérez-García et al., 2001). Heteroflorum (Leguminosae), género nuevo para la ciencia aún no publicado y colectado por primera vez en la cuenca del Río Balsas (M. Sousa, com. pers.), fue encontrado en Zimatán durante las exploraciones botánicas de este trabajo, ampliando así su distribución.

La familia Leguminosae, por el número de especies con que cuenta, es la mejor representada en el área de estudio, con varios géneros (Acacia, Desmodium, Indigofera, Lonchocarpus, Mimosa y Senna) que se cuentan entre los que tienen el mayor número de especies en la región. Le sigue en importancia la familia Asteraceae, con el género Eupatorium con el mayor número de especies. La tercer familia mejor representada es Poaceae, pero ninguno de sus géneros se encuentra entre los de mayor riqueza específica. Es notable la abundancia del género Solanum (18 especies), mientras que los cinco géneros restantes de esta familia sólo están representados por una especie. El género Cordia destaca con 16 especies, una de las cuales es nueva para la ciencia. Con base en el número de especies de la familia Leguminosae, se espera tener aproximadamente 2,200 especies en la flora total de la región de Zimatán (M. Sousa, com. pers.).

Una de las intenciones de generar información florística básica de la región de Zimatán era ubicar especies que tuvieran algún status de conservación o que fueran nuevas para la ciencia, lo que permitiría dar un valor adicional a la conservación del área. Con la lista florística actual se han

Cuadro 3. Especies listadas en la NOM-059ECOL-2001 presentes en la región de Zimatán.

\begin{tabular}{|c|c|c|}
\hline Nombre científico (familia) & Nombre común & Status \\
\hline Albizia plurijuga (Leguminosae) & & Amenazada \\
\hline Astronium graveolens (Anacardiaceae) & Sangualico & Amenazada \\
\hline Bravaisia integerrima (Acanthaceae) & Sanate & Amenazada \\
\hline Chamaedorea pochutlensis (Arecaceae) & & Amenazada \\
\hline Cryosophila nana (Arecaceae) & Salmiche & Amenazada \\
\hline Epidendrum cnemidophorum (Orchidaceae) & & Amenazada \\
\hline Licania arborea (Chrysobalanaceae) & Carnero & Amenazada \\
\hline Sapium macrocarpum (Euphorbiaceae) & Palo de leche & Amenazada \\
\hline Tabebuia chrysantha (Bignoniaceae) & Cortés & Amenazada \\
\hline Tillandsia concolor (Bromeliaceae) & Gallito & Amenazada \\
\hline Peniocereus fosterianus (Cactaceae) & & Rara \\
\hline Zamia paucijuga (Zamiaceae) & Palmita & Rara \\
\hline Avicennia germinans (Verbenaceae) & Mangle & Protección especial \\
\hline Conocarpus erectus (Combretaceae) & Mangle salado & Protección especial \\
\hline Guaiacum coulteri (Zygophyllaceae) & Guayacán & Protección especial \\
\hline Laguncularia racemosa (Combretaceae) & Mangle & Protección especial \\
\hline Saurauia serrata (Actinidiaceae) & & Protección especial \\
\hline Tigridia orthantha (Iridaceae) & & Protección especial \\
\hline Dalbergia congestiflora (Leguminosae) & Granadillo & Peligro de extinción \\
\hline Dalbergia granadillo (Leguminosae) & Granadillo & Peligro de extinción \\
\hline
\end{tabular}


detectado 20 especies incluidas en la NOM-059ECOL-2001 (cuadro 3). Hay varias especies de zonas húmedas y manglares que están siendo eliminadas principalmente para sustituir la vegetación original por tierras agrícolas. Algunas especies, como Dalbergia granadillo y D. congestiflora, que se encuentran en muy bajas densidades en estas selvas, son frecuentemente buscadas por su madera. Bourreria rekoi, especie colectada y descrita a principios de siglo pasado, no había sido vuelta a colectar. Durante el desarrollo de este proyecto fue localizada en lugares diferentes a la localidad tipo y se colectó por primera vez el fruto, el cual era aún desconocido para la ciencia. Con base en observaciones de campo se propone que Bourreria rekoi sea considerada como especie rara, de acuerdo a la nomenclatura utilizada en la NOM-059ECOL-2001, hasta que haya mayor información que determine su vulnerabilidad.

Vegetación. De acuerdo a la clasificación de vegetación de Miranda y Hernández-X. (1963), se encuentran los siguientes tipos: los alrededores de las lagunas con agua salobre están bordeadas por manglares, que en el área son más bien escasos y colindan tierra adentro con selva baja espinosa caducifolia; a su vez, ésta da lugar a selvas bajas y medianas caducifolias. Estas dos últimas son los tipos de vegetación que cubren la mayor superficie en la región de estudio; un cálculo aproximado es de 50,000 ha (García et al., 1992). Hacia mayores altitudes y en las vegas de los ríos se encuentran selvas medianas subcaducifolias y subperennifolias. Finalmente, en las altitudes máximas (1,800 a 2,600 m s.n.m.) se desarrollan los bosques mesófilos de montaña y bosques templados de pino-encino. En pequeñas áreas dispersas, localizadas en el norte de la región, se encuentran sabanas y encinares, que colindan generalmente con selvas medianas subcaducifolias y subperennifolias. A continuación se presenta una descripción breve para los tipos de vegetación encontrados.

Vegetación de dunas costeras.- Esta vegetación se desarrolla en una franja angosta a la orilla del mar, por lo que puede estar expuesta al agua salina durante las mareas altas; está dominada por plantas herbáceas, entre las que destacan Paspalum vaginatum, Pectis haenkeana, Ipomoea pescaprae, Batis maritima y Okenia hypogaea.

Manglares.- En los alrededores de las lagunas con agua salobre y de las desembocaduras de los ríos se encuentran manglares dominados por Avicennia germinans, Conocarpus erecta y Laguncularia racemosa.

Selva baja espinosa caducifolia.- Colindando con las dunas costeras, en sustrato todavía arenoso, se presentan selvas bajas espinosas caducifolias, cuyos integrantes no rebasan los $3 \mathrm{~m}$ de altura. Su composición florística está dominada por especies espinosas, como Acacia cochliacantha, A. farnesiana, A. cornigera y Ziziphus amole, además de varias especies de cactos como Pachycereus pecten-aboriginum, Pilosocereus collinsii, Pereskiopsis diguetii, Opuntia decumbens y Pereskia lychnidiflora, las cuales están acompañadas por Bursera excelsa, B. laurihuertae, Guaiacum coulteri, Amphipterygium adstringens, Caesalpinia coccinea, Capparis flexuosa, Forchammeria pallida y Cordia truncatifolia, entre otras.

Selva mediana y baja caducifolia.- En extensas superficies de esta región, las selvas caducifolias presentan una estructura que no corresponde estrictamente con la descripción que hacen Miranda y Hernández-X. (1963) de ellas específicamente por su altura, ya que los árboles alcanzan hasta $25 \mathrm{~m}$. Al respecto, Salas-Morales (2002) propone que debido a su altura, éstas sean consideradas como selvas medianas caducifolias. Estas selvas se desarrollan en un intrincado mosaico junto con comunidades que no rebasan $15 \mathrm{~m}$ de altura, las cuales corresponderían según Miranda y Hernández-X. (1963), a las selvas bajas caducifolias. Ambas pierden sus hojas durante la época seca del año y florísticamente son muy similares. Estructuralmente, las selvas medianas caducifolias se distinguen por presentar dos estratos arbóreos, mientras que en las selvas bajas sólo se distingue uno. El estrato arbóreo alto de las selvas medianas cuenta con algunas especies que lo caracterizan, tales como Lonchocarpus aff. magallanesii, Euphorbia calyculata, Gyrocarpus americanus, Cordia sonorae y C. tinifolia. Entre las especies que integran indistintamente ambos estratos se pueden mencionar a Albizia occidentalis, Amphipterygium adstringens, Bucida macrostachya, Bursera excelsa, B. graveolens, B. heteresthes, B. simaruba, Cochlospermum vitifolium, Comocladia engleriana, Cordia elaeagnoides, Guaiacum coulteri, Jacaratia mexicana, Lonchocarpus constrictus, L. emarginatus, Morisonia americana, Tabebuia chrysantha y Pterocarpus acapulcensis.

Selva mediana subcaducifolia y subperennifolia.- En las estribaciones de la Sierra Madre del Sur y en las vegas de los ríos hay selvas medianas subcaducifolias con Andira inermis, Astianthus viminalis, Astronium graveolens, Enterolobium cyclocarpum, Hura polyandra, Inga vera, I. paterno, Pithecellobium dulce, P. lanceolatum, Swietenia humilis, Thouinidium decandrum, etc. En contraste, a mayor altitud, en laderas con exposición norte en sitios con mayor disposición de humedad, se desarrollan selvas medianas subperennifolias con Bernoullia flammea, Bourreria rekoi, Brosimum alicastrum, Cojoba arborea, Castilla elastica, Cupania dentata, Hymenaea courbaril, Poulsenia armata, etc. Los árboles de estas selvas son utilizados como sombra para el cultivo del café, que es una de las principales actividades de la economía regional (García et al., 1992).

Sabana.- Las sabanas se localizan en el norte del área, en superficies muy pequeñas y de manera dispersa en las faldas del Cerro de la Virgen y en una gran superficie continua en el norte de San Miguel Chongo. A diferencia de las sabanas de Nizanda en el Istmo de Tehuantepec, dominadas por hierbas amacolladas (Pérez-García et al., 2001), en Zimatán existe un estrato arbóreo bajo de no más de $3 \mathrm{~m}$ de altura, con 
Curatella americana, Byrsonima crassifolia, Psidium guajava, Bursera bipinnata e Hymenaea courbaril, con algunas eminencias de mayor altura de Pinus maximinoi. En ellas se distingue un estrato arbustivo y el estrato herbáceo está dominado principalmente por las familias Poaceae, Cyperaceae y Asteraceae.

Encinares.- Los encinares se distribuyen en pequeñas áreas de forma discontinua en el norte de la región. Su estrato arbóreo está caracterizado por una gran abundancia de encinos, sobre todo Quercus conspersa, Q. glaucescens, Q. magnoliifolia y $Q$. peduncularis. Están frecuentemente acompañadas por especies de las selvas húmedas que rodean a este tipo de vegetación, como Trophis racemosa, Agonandra racemosa, Godmania aesculifolia y varias especies de Ficus.

Uso de suelo. Grandes superficies de la región han sido deforestadas con fines principalmente agrícolas. En las vegas de los ríos y los terrenos planos, se cultivan principalmente papaya, limón y plátano. La agricultura de temporal de maíz y frijol ha desplazado cientos de hectáreas de selvas en diversos lugares del área, incluyendo laderas con pendientes muy pronunciadas. Este proceso se ha agudizado en los últimos años con los programas de incentivos para el desarrollo rural. La ganadería que se practica es de tipo extensivo, dejando pastar al ganado libremente entre los árboles de las selvas, por lo que grandes superficies de selva han sido alteradas por esta actividad.

\section{Conclusiones}

No se conoce ninguna investigación florística previa para el área de estudio ni sus alrededores, por lo que este trabajo presenta los primeros resultados sobre la composición florística y la vegetación de una porción de las regiones Costa y Sierra Sur de Oaxaca; ambas fueron reportadas por GarcíaMendoza y Torres-Colín (1999) como pobremente colectadas.

La diversidad de los tipos de vegetación presentes en la región de Zimatán en un transecto longitudinal relativamente corto se manifiesta en su riqueza florística. Con una superficie ligeramente superior a $700 \mathrm{~km}^{2}$, Zimatán rebasa los valores de riqueza de especies publicado para las floras de otras regiones cuyas superficies son mucho mayores. Es posible que estas diferencias se deban, al menos en parte, a que dichos estudios se basan en menores números de colecta, inclusive considerando los registros consultados en herbarios (cuadro 4).

El hallazgo de especies cuya distribución se limitaba a Centro y Sudamérica (p.ej. Gyrocarpus americanus, Lophostachys guatemalensis var. nov. y Aristolochia anguicida) muestra que la flora de Zimatán se compone no sólo por los elementos propios de la vertiente del Pacífico mexicano, sino que su ubicación geográfica le permite el contacto con otras regiones, dando por resultado una gran riqueza florística. Aparentemente existen varias especies más que no habían sido registradas para México o para Oaxaca, lo que muestra la falta de conocimiento que se tenía del área estudiada. Sin embargo, éstas se revisarán taxonómicamente con el fin de confirmar su identidad.

El esfuerzo de colecta se ha concentrado principalmente en las selvas bajas y medianas caducifolias, por lo que se considera que los tipos de vegetación presentes a partir de $600 \mathrm{~m}$ de altura no están suficientemente conocidos florísticamente. Por ello, los trabajos futuros de colecta deben estar enfocados a esta parte del área.

Las selvas medianas subperennifolias han sido tradicionalmente utilizadas para el cultivo del café. Afortunadamente y a diferencia de otras áreas, el dosel arbóreo que se ha usado para su sombra ha sido el de los árboles nativos; por lo tanto, aún se pueden encontrar novedades botánicas y redescubrir especies colectadas a principios del siglo pasado, como fue el caso de Bourreria rekoi. Se sabe poco de la composición florística de este tipo de vegetación, pero se conoce mucho menos de su estructura, diversidad, distribución y estado de conservación. Es importante dirigir esfuerzos en esta dirección, que se tornan

Cuadro 4. Comparación de la riqueza de las floras de otras regiones con la de Zimatán.

\begin{tabular}{|c|c|c|c|c|c|}
\hline Región & $\begin{array}{l}\text { Superficie } \\
\left(\mathrm{km}^{2}\right)\end{array}$ & No. de familias & No. de géneros & No. de especies & No. de Registros \\
\hline $\begin{array}{l}\text { Depresión Central, Chiapas } \\
\text { (Reyes-García y Sousa, 1997) }\end{array}$ & 9,000 & 103 & 489 & 998 & 1,261 \\
\hline $\begin{array}{l}\text { Distrito de Tehuantepec, Oaxaca } \\
\text { (Torres-Colín et al., 1997) }\end{array}$ & 6,600 & 154 & 776 & 1,720 & 8,500 \\
\hline $\begin{array}{l}\text { Costa Grande, Guerrero } \\
\text { (Peralta-Gómez et al., 2000) }\end{array}$ & 2,500 & 135 & 527 & 1,047 & 3,020 \\
\hline Región de Zimatán, Oaxaca & 713 & 144 & 668 & 1,384 & 8,063 \\
\hline
\end{tabular}


urgentes ante la caída de los precios del café, lo cual puede llegar a repercutir sobre la permanencia del cultivo y por ende de las selvas medianas subperennifolias.

Las diferencias encontradas en la fisonomía y la composición florística de las sabanas del área de estudio en contraste con las de Nizanda, plantean una línea de investigación que conduzca a determinar la magnitud y las causas de estas disimilitudes en un mismo tipo de vegetación.

Con referencia a las especies presentes en el área de estudio y listadas en la NOM-059-ECOL-2001, según nuestra experiencia de campo, su vulnerabilidad se origina por diversas causas. Algunas de ellas se encuentran en riesgo por la eliminación del tipo de vegetación que integran, p.ej. Astronium graveolens, Bravaisia integerrima, Avicennia germinans o Conocarpus erectus; otras son muy apreciadas por la calidad de su madera, por lo que han sido extraídas de varias áreas (p.ej. Dalbergia granadillo), mientras que otras tienen, de manera natural, bajas densidades en estas selvas (p.ej. Sapium macrocarpum y Zamia paucijuga).

Finalmente, se considera que la información florística que se presenta es suficiente para concluir que Zimatán es una región sumamente importante, no sólo por la diversidad de tipos de vegetación, sino por su riqueza específica y por la presencia de especies en riesgo y de otras nuevas para la ciencia. Además, representa una de las áreas más extensas de selvas secas bien conservadas en la costa de Oaxaca y, hasta donde sabemos, la única selva mediana caducifolia que aún existe en México. El acelerado cambio del uso de suelo que está teniendo lugar en esta región oaxaqueña, transformando grandes superficies de selvas en pastizales y áreas agrícolas, plantea una urgente necesidad de tomar medidas que permitan conservar los ecosistemas y organismos aún presentes, pero a la vez hacer un uso adecuado de ellos, esto es, que sea compatible con su permanencia.

\section{Agradecimientos}

En el desarrollo del presente trabajo fue fundamental el apoyo del Dr. Mario Sousa Sánchez y el personal del Herbario Nacional (MEXU). Queremos agradecer la colaboración de los siguientes especialistas: Salvador Acosta (Acanthaceae), Frank Almeda (Melastomataceae), Gloria Andrade (Leguminosae), Salvador Arias (Cactaceae), Daniel F. Austin (Convolvulaceae), María Goreti Campos (Boraginaceae), Javier Castrejón Reyna (Cucurbitaceae), Gerrit Davidse (Poaceae), Alfonso Delgado (Leguminosae), Nelly Diego (Cyperaceae), Robert L. Dressler (Orchidaceae), Adolfo Espejo (Commelinaceae, Bromeliaceae), Gabriel Flores (Leguminosae), Juan Carlos Flores (Moraceae, Arecaceae), Abisaí García (Agavaceae), María Teresa Germán (Meliaceae), Rosaura Grether (Leguminosae), Colin Hughes (Leguminosae), Guillermo Ibarra (Moraceae), Jaime Jiménez (Euphorbiaceae), Rolando Jiménez (Orchidaceae), Verónica Juárez (Asclepiadaceae), Lawrence Kelly (Aristolochiaceae),
Rafael Lira (Cucurbitaceae), Francisco Lorea (Lauraceae), Lucio Lozada (Asclepiadaceae), Martha Martínez (Euphorbiaceae), Esteban Martínez (Bignoniaceae y otras), John Mickel (Pteridophyta), J. Carlos Montero (Solanaceae), Michael Nee (Solanaceae), Kevin Nixon (Fagaceae), Helga Ochoterena (Rubiaceae), Miguel A. Pérez F. (Araceae), J. A. Pérez de la Rosa (Pinaceae), Francisco Ramos (Asteraceae y otras), Clara Ramos (Rutaceae y otras), Jerónimo Reyes (Cactaceae), Lourdes Rico (Leguminosae), Jerzy Rzedowski (Burseraceae), Gerardo Salazar (Orchidaceae), Stephen L. Solheim (Sterculiaceae), Miguel A. Soto (Orchidaceae), Mario Sousa (Leguminosae), Charlotte Taylor (Rubiaceae), Oswaldo Téllez (Dioscoreaceae), Rafael Torres (Leguminosae), Leticia Torres (Leguminosae), K. B. Utley (Begoniaceae), Susana Valencia (Fagaceae), Mario Veliz (Bromeliaceae), José Luis Villaseñor (Asteraceae). El Dr. Jorge Meave, el M. en C. Eduardo Pérez-García, el Dr. José Luis Villaseñor y un revisor anónimo hicieron valiosas observaciones y sugerencias al manuscrito. Alice Boyle nos apoyó con el resumen en inglés y Arturo Zafra en el arreglo del listado florístico.

Misael Elorza Castillo, José Pascual y Feliciano López apoyaron en las colectas botánicas. Un agradecimiento a todas las personas que colaboraron en las diferentes etapas de este trabajo: Javier Castrejón Reyna, Erika M. Lira, Esteban Martínez Salas, Catherine Perret, Jaime E. Rivera Hernández, Arturo Sánchez, Ricardo de Santiago, Elizabeth Torres Bahena y Noé Velázquez Rosas, y a la gente sin cuya ayuda hubiera sido muy difícil nuestro trabajo: José Luis Márquez Aguilar, Nancy Salas Morales, Rosenda Borja y Lluvia Ricárdez. El apoyo financiero para llevar a cabo este estudio fue otorgado por las siguientes instituciones: Fundación MacArthur a través del convenio 99-57144-G55; Fondo Mundial para la Naturaleza (WWF) oficina Oaxaca a través de los convenios PN06, PN94 y PP68; Fondo Mexicano para la Conservación de la Naturaleza con el convenio B-198/ 015 y del Sistema de Investigación Benito Juárez (SIBEJCONACyT) con el convenio 19990506007. A todas ellas un profundo agradecimiento.

\section{Literatura citada}

Arriaga L., Espinoza J.M., Aguilar C., Martínez E., Gómez L. y Loa E. Coordinadores. 2000. Regiones Terrestres Prioritarias de México. Comisión Nacional para el Conocimiento y Uso de la Biodiversidad, México, D.F.

Brummitt R.K. y Powell C.E. Eds.. 1992. Authors of Plant Names. The Royal Botanic Gardens, Kew.

CETENAL-UNAM. 1970. Carta de Climas San Pedro Pochutla 14P(II) Oaxaca 14Q-VIII. México, D.F.

Cronquist A. 1981. An Integrated System of Classification of Flowering Plants. Columbia University Press, Nueva York.

Diario Oficial de la Federación. 2002. Norma Oficial Mexicana NOM-059-ECOL-2001, Protección ambiental- Especies nativas de México de flora y fauna silvestres- Categorías de riesgo y 
especificaciones para su inclusión, exclusión o cambio- Lista de especies en riesgo. Segunda Sección, 6 de marzo de 2002, $1-56$.

Ferrusquía-Villafranca I. 1998. Geología de México: una sinopsis. En: Ramamoorthy T., Bye R., Lot A. y Fa J. Comps. Diversidad Biológica de México: Orígenes y Distribución, pp. 3-108, Instituto de Biología, Universidad Nacional Autónoma de México, México, D.F.

García E. 1988. Modificaciones al Sistema de Clasificación Climática de Köppen. Cuarta edición. Editado por la autora, México, D.F.

García G., Salas S.H., Schibli L., Aguilar R., Acosta S. y Salazar A. 1992. Análisis de la vegetación y uso actual del suelo en el estado de Oaxaca, Fase I (Costa y Sierra Sur). Informe Técnico presentado a WWF. SERBO, A.C. Oaxaca, Oax.

García-Mendoza A. y Torres-Colín R. 1999. Estado actual del conocimiento sobre la flora de Oaxaca. En: Vásquez-Dávila M.A. Ed. Sociedad y Naturaleza en Oaxaca 3: Vegetación y Flora, pp. 50-86, Instituto Tecnológico Agropecuario de Oaxaca, Oaxaca, Oax.

INEGI (Instituto Nacional de Estadística, Geografía e Informática). 1980. Carta de Climas Villahermosa. Escala 1:1,000,000. México, D.F.

INEGI. 1984. Carta Geológica E15-10 D15-1 Juchitán. Escala 1:250,000. México, D.F.

INEGI. 1988a. Carta Geológica D14-3 Puerto Escondido. Escala 1:250,000. México, D.F.

INEGI. 1988b. Carta Edafológica E15-10 D15-1 Juchitán, provisional. Escala 1:250,000. México, D.F.

INEGI. 1989. Carta Edafológica D14-3 Puerto Escondido. Escala 1:250,000. México, D.F.

Lorence D.H. y García-Mendoza A. 1989. Oaxaca, México. En: Campbell D.G. y Hammond H.D. Eds. Floristic Inventory of Tropical Countries: the Status of Plant Systematics, Collections, and Vegetation, plus Recommendations for the Future, pp. 253269, New York Botanical Garden, Nueva York.

Lott E. 1985. Listados Florísticos de México III. La Estación de Biología de Chamela, Jalisco. Instituto de Biología, Universidad Nacional Autónoma de México, México, D.F.

Mickel J.T. y Beitel J.M. 1988. Pteridophyte Flora of Oaxaca,
Mexico. Memoirs of the New York Botanical Garden 46:1-568. Miranda F. y Hernández-X. E. 1963. Los tipos de vegetación de México y su clasificación. Boletín de la Sociedad Botánica de México 28:29-179.

Peralta-Gómez S., Diego N. y Gual-Díaz M. 2000. Listados Florísticos de México XIX. La Costa Grande de Guerrero. Instituto de Biología, Universidad Nacional Autónoma de México, México, D.F.

Pérez-García E.A., Meave J. y Gallardo C. 2001. Vegetación y flora de la región de Nizanda, Istmo de Tehuantepec, Oaxaca, México. Acta Botanica Mexicana 56:19-88.

Reyes-García A. y Sousa M. 1997. Listados Florísticos de México XVII. Depresión Central de Chiapas. La Selva Baja Caducifolia. Instituto de Biología, Universidad Nacional Autónoma de México, México, D.F.

Rzedowski J. 1978. Vegetación de México. Limusa, México, D.F.

Rzedowski J. 1991. Diversidad y orígenes de la flora fanerogámica de México. Acta Botanica Mexicana 14:3-21.

Salas-Morales S.H. 2002. Relación entre la heterogeneidad ambiental y la variabilidad estructural de las selvas tropicales secas de la costa de Oaxaca, México. Tesis de Maestría, Facultad de Ciencias, Universidad Nacional Autónoma de México, México, D.F., 101 pp.

Toledo V.M. 1988. La diversidad biológica de México. Ciencia y Desarrollo 14:17-30.

Torres-Colín M.L. 1989. Estudio florístico y descripción de la vegetación del Cerro Guiengola, en el Istmo de Tehuantepec, Oaxaca. Tesis de Licenciatura, Escuela Nacional de Estudios Profesionales Iztacala, Universidad Nacional Autónoma de México, Los Reyes Iztacala, Edo. Méx., 81 pp.

Torres-Colín R., Torres-Colín L., Dávila-Aranda P. y VillaseñorRíos J.L. 1997. Listados Florísticos de México XVI. Flora del Distrito de Tehuantepec, Oaxaca. Instituto de Biología, Universidad Nacional Autónoma de México, México, D.F.

Zizumbo-Villarreal D. y Colunga G.P. 1980. La utilización de los recursos naturales entre los huaves de San Mateo del Mar, Oaxaca. Tesis de Licenciatura, Facultad de Ciencias, Universidad Nacional Autónoma de México, México, D.F. 375 pp.

Fecha de recepción: 26 de marzo de 2003

Versión corregida: 21 de mayo de 2003

Aceptado: 21 de mayo de 2003 
Apéndice 1. Lista de plantas vasculares de la Costa de Oaxaca: región de Zimatán.

Los números de colecta que se incluyen en la lista florística pertenecen a personas que colaboraron en algún momento para el proyecto de la flora de la costa de Oaxaca. A continuación se presentan en orden alfabético los nombres completos y las abreviaturas que se utilizaron para cada uno de ellos. ASA Alberto Sánchez, CH Colin Hughes, CP Catherine Perret, DS Debora Scheideger, EM Esteban Martínez Salas, ET Elizabeth Torres Bahena, FL Feliciano López Agustín, JFC Javier Castrejón Reyna, JP José Pascual Cortez, JR Jaime Rivera Hernández, LS Leo Schibli, MC Miguel Cerón Medina, ME Misael Elorza Castillo, NV Noé Velázquez Rosas, RdeS Ricardo de Santiago, RGS Rafael García Soriano, SAY Alfredo Saynes Vásquez, SS Silvia H. Salas Morales, YAC Yaayé Arellanes Cancino. Las especies marcadas con $¥$ son introducidas o cultivadas.

\section{PTERIDOPHYTA}

\section{ADIANTACEAE}

Acrostichum aureum L.

ME1160.

Adiantopsis radiata (L.) Fée

JR1803.

Adiantopsis seemannii (Hook.) Maxon

JR 1732.

Adiantum amblyopteridium Mickel et Beitel

FL 74, SAY 1971.

Adiantum amplum C.Presl

SS 2697, 2797, 3058, 3861.

Adiantum capillus-veneris L.

FL 6, SS 3858.

Adiantum concinnum Humb. et Bonpl. ex Willd.

CP 734, 774, EM 33093, JFC 903,1074, JR 1800, SS 2521, 2709.

Adiantum patens Willd.

SAY 2287.

Adiantum philippense L.

JR 1796, SS 2520.

Adiantum princeps T.Moore

EM 32930, JR 1767, 1799.

Adiantum trapeziforme L.

FL 9, 71, JR 1770, SAY 1961, 2268, SS 2711, 2800, 3675, 3863.

Adiantum villosum $\mathrm{L}$.

JR 1768.

Bommeria pedata (Sw.) E.Fourn.

SAY 2293.

Cheilanthes affinis Mett.

JFC 549.

Cheilanthes angustifolia Kunth

SAY 2292.

Cheilanthes brachypus (Kunze) Kunze

JR 825, SS 2983, 2986.

Cheilanthes chaerophylla (M.Martens et Galeotti) Kunze

SAY 2290.

Cheilanthes mickelii T.Reeves

SS 1848.

Cheilanthes rigida (Sw.) Mett.

JR 1738.

Cheilanthes skinneri (Hook.) T.Moore

JR 1584.

Hemionitis pinnatifida Baker

JFC 745, SS 3513.

Pityrogramma calomelanos (L.) Link

CP 740, 761, 775, SS 2851, 3484.
Pteris grandifolia L.

JFC 879, JR 1754.

Pteris longifolia L.

CP 730, ME 2150.

ASPLENIACEAE

Asplenium hallbergii Mickel et Beitel FL 109a.

Asplenium potosinum Hieron.

FL 72.

Ctenitis equestris (Kunze) Ching

SAY 2291.

Polystichum speciosissimum (A.Braun ex Kunze) Copel. FL 89.

Tectaria heracleifolia (Willd.) Underw. CP 770, JR 1765, 2406, SAY 1985, SS 2799, 3855.

Tectaria mexicana (Fée) C.V.Morton CP 742, FL 73, 322, SS 3859, 3860.

BLECHNACEAE

Blechnum occidentale L. SAY 1977, 2273, SS 2710, 2735.

EQUISETACEAE

Equisetum sp. JR 2442.

LOPHOSORIACEAE

Lophosoria quadripinnata (J.F.Gmel.) C.Chr. SAY 1992.

POLYPODIACEAE

Niphidium crassifolium (L.) Lellinger JR 2415.

Pecluma alfredii (Rosenst.) M.G.Price JR 2451.

Pleopeltis sp. FL 105.

Polypodium sp. FL 122.

SCHIZAEACEAE

Anemia hirsuta (L.) Sw. SS 3003.

Anemia oblongifolia (Cav.) Sw. EM 32934, NV 312, SS 3356.

Anemia pastinacaria Moritz ex Prantl JFC 919, 928, SS 3074. 
Anemia phyllitidis (L.) Sw.

SS 3532.

Lygodium venustum Sw.

JFC 687, SS 3350.

\section{SELAGINELLACEAE}

Selaginella delicatissima Linden ex A.Braun

JFC 887.

Selaginella hoffmannii Hieron.

JR 1733.

Selaginella pallescens (C.Presl) Spring

JR 1787, 1802, NV 313.

Selaginella sertata Spring

SS 3123.

THELYPTERIDACEAE

Thelypteris hispidula (Decne.) C.F.Reed

ME 1831.

Thelypteris imbricata (Liebm.) C.F.Reed

SAY 2271.

Thelypteris torresiana (Gaudich.) Alston

JR 1766.

\section{CYCADOPHYTA}

\section{ZAMIACEAE}

Zamia paucijuga Wieland MC 388.

\section{CONIFEROPHYTA}

\section{PINACEAE}

Pinus maximinoi H.E.Moore

SS 2769, 4271, SAY 23

\section{MAGNOLIOPHYTA}

MAGNOLIOPSIDA

\section{ACANTHACEAE}

Anisacanthus quadrifidus (Vahl) Nees

SAY 2818.

Aphelandra gigantiflora Lindau

JP 223, SAY 2737.

Aphelandra scabra (Vahl) Sm.

CP 185, 658, FL 404, 430, JP 139, 247, JR 2132, ME 1032,

$1375,1507,1803,5880$, SAY $2781,2789,2815,2903$, SS

2003, 2599, 3623, 3635.

Barleria oenotheroides Dum.Cours.

JR 2176, SAY 2902, SS 3630, 3697.

Blechum grandiflorum Oerst.

SS 3651.

Blechum pyramidatum (Lam.) Urb.

EM 32200, 32201, ME 2001, 2912, 4192, 4335, SAY 2040,

SS 1294, 2820, 2848, 3847.

Bravaisia integerrima (Spreng.) Standl.

CH 443, 1931, EM 32169, JR 401, ME 175, 1989, 1994, 2075, SAY 2871.

Carlowrightia arizonica A.Gray

CP 166, 173, 214, JR 345, ME 4161, 4450, 5521, 5656a,

SAY 2812, SS 1975, 2022, 3459a.
Elytraria imbricata (Vahl) Pers.

CP 163, 218, JFC 536, 1083, ME 1872, 2452, 2913, 4076, 4079, SS 1293, 2020, 2625, 3417.

Henrya insularis Nees

CP 212, DS 81, EM 32058, 32314, FL 63, JP 316, 365, 367, ME 72, 1778, 2839, 5656b, 5907, SAY 2077, 2901, SS 2012, 3634, 3870.

₹ Hypoestes phyllostachya Baker SS 2713.

Jacobinia mexicana Seem. ME 1125,1647, SS 1972.

Jacobinia mollis Greenm. ME 3886.

Justicia alopecuroidea T.F.Daniel SS 3650.

Justicia aurea Schltdl. SAY 1976, SS 3659.

Justicia candicans (Ness) L.D.Benson CP 463, EM 32449, ME 1066, 3728, 5233, 5519, SS 2486. Justicia carthaginensis Jacq. JR 953, ME 831.

Justicia furcata Jacq. JP 138.

Justicia ramosa (Oerst.) V.A.W.Graham CP 169, 190, JFC 537, ME 5517, SS 2021.

Justicia aff. santelisiana Acosta et T.F. Daniel ME 1447.

Justicia sp. nov. ME 2481, 5520.

Lophostachys guatemalensis Donn.Sm. var. nov. JP 332, JR 2174, SS 2839.

Ruellia conzattiana Standl. FL 399.

Ruellia foetida Willd. CP 189, 215, EM 32089, JP 244, 242, JR 553, ME 1245 , 1371, 3868, 4077, SS 3462, 3519.

Ruellia hookeriana (Nees) Hemsl. ME 1804.

Ruellia inundata Kunth CP 171, 188, 210, JFC 618, ME 1616, 2560, 2567, 2651, SS 2622, 3451, 3507, 3788.

Ruellia paniculata L. ME 2531, 2829, 5790, 5891, RGS 384.

Ruellia pringlei Fernald ME 3852.

Ruellia speciosa Mart. ex Nees FL 87, SS 3649, 3912.

Siphonoglossa sp. JR 1084, ME 1649, 1873.

Tetramerium nervosum Nees CP 172, JFC 535, 544, JP 134, ME 84, 5482, SS 2033, 2560, 2632, 3459b.

\section{ACHATOCARPACEAE}

Achatocarpus gracilis $\mathrm{H}$.Walter EM 32166, JFC 859, 860, ME 1364, 1420, 1533, 2529, SS 1971.

Achatocarpus oaxacanus Standl. CH 1929, JR 2100, 394, ME 566, 1298, 1493, 5658, RGS 396, SS 1915. 
ACTINIDIACEAE

Saurauia serrata DC.

SS 2721.

Saurauia villosa DC.

MC 358.

\section{AIZOACEAE}

Trianthema portulacastrum L.

EM 32177, ME 1844, 1992.

\section{AMARANTHACEAE}

Achyranthes sp.

NV 351.

Alternanthera flava (L.) Mears

ME 3731.

Alternanthera gracilis (M.Martens et Galeotti) Loes.

ME 1449.

Amaranthus palmeri S.Watson

EM 32220, ME 35, 819, 1019, 1746.

Amaranthus spinosus L.

ME 1321, 2008, 4998, 5100.

Celosia nitida Vahl

JR 1052.

Chamissoa acuminata Mart. var. swansonii Sohmer

CP 178, ME 76.

Chamissoa altissima (Jacq.) Kunth

ME 1607.

Gomphrena decumbens Jacq.

NV 278, SS 3200, 3208.

Iresine calea (Ibáñez) Standl.

CP 216, ME 3878.

Iresine diffusa Humb. et Bonpl. ex Willd.

FL 440.

\section{ANACARDIACEAE}

Anacardium occidentale L.

SS 2808.

Astronium graveolens Jacq.

JR 897, 2196, ME 460.

Comocladia engleriana Loes.

ET 1278, JR 1078, 2199, 2203, MC 363, SS 1284.

₹ Mangifera indica L.

DS 66.

Spondias mombin L.

ME 310.

Spondias purpurea L.

CH 1932, CP 192, DS 27, 104, EM 32050, ET 1326, JR 472,2168, 2443, ME 1482, 1586, 1656, 1823, RdeS 712, SAY 2488, SS 1570, 1997, 2320.

\section{ANNONACEAE}

Mosannona depressa (Baill.) Chatrou

JR 2503, SS 2817.

Rollinia membranacea Triana et Planch.

FL 42, JR 2470.

Sapranthus violaceus (Dunal) Saff.

CH 1916, CP 177, ME 93, 1053, 2195, 2443.

\section{APIACEAE}

Hydrocotyle verticillata Thunb. var. triradiata (A.Rich.) Fernald.

EM 32081, JFC 805, ME 985, 1738, 2005.
Spananthe paniculata Jacq.

JFC 642.

\section{APOCYNACEAE}

Aspidosperma megalocarpon Müll.Arg.

FL 218, JR 1042, ME 1986, 2132, 2874.

Cascabela ovata (Cav.) Lippold

CH 1940, CP 26, 309, JFC 861, JP 142, JR 415, 717, 734, 780, MC 383, ME 154, 167, 2020, 2086, SAY 2412, 2674, SS 1273, 3216, 3217, YAC 164.

Cascabela thevetioides (Kunth) Lippold

CT 459a, ME 211.

Catharanthus rosea (L.) G.Don

JR 331.

Echites yucatanensis Millsp. ex Standl.

JFC 735, ME 2331, 3360, 3486.

Fernaldia pandurata (A.DC.) Woodson

JP 13, 1039, 1748, 1900, ME 390, 1146, 2360, 3876, 5434, SS 1533.

Haplophyton cimicidum A.DC.

CP 73, 184, 527, 622, 668, FL 135, JR 821, 905, 1598, 2383, ME 1009, 2250, 3172 SS 1508, 2997,4219.

Laubertia contorta (M.Martens et Galeotti) Woodson JP 32, ME 2279.

Mandevilla subsagittata (Ruiz et Pav.) Woodson FL 211, JP 115, JR 1747, 1880, 2486, NV 429, 505, 506, 519, SAY 2583, 2600, SS 1844, 3327, 3358.

Mandevilla subsessilis (A.DC.) Woodson JR 1880.

Pentalinon andrieuxii (Müll.Arg.) B.F.Hansen et Wunderlin CT 446, 464bis, 465, JFC 837, JR 2560, ME 1110, 1186, 1365, 1526.

Plumeria rubra L.

CT 418, JP 439, JR 2308, 2346, ME 169, 2079, 2327, 2792, SS 1764.

Prestonia mexicana A.DC.

ME 1812.

Rauvolfia tetraphylla L.

ASA 75, CT 438, EM 32197, 32368, FL 187, 286, JR 346, 440, 453, 755, ME 1233, 1612, 1713, 1937, SS 2647, YAC 153.

Stemmadenia macrophylla Greenm.

FL 33, 85.

Stemmadenia obovata (Hook. et Arn.) K.Schum. ASA 74, CP 307, CT 430, 453, 456bis, EM 32075, JP 449, JR 344, 720, 1083, ME 160, 168, 1862, 2050, , RGS 408, SS 1586, 1611, 2025, 2054, 2137, 3498, YAC 135.

Tabernaemontana amygdalifolia Jacq. CT 487bis, EM 32061, JP 447, ME 1099, 1767, 1900, 2116, RGS 403, SS 1774, 1796, 1960.

Thevetia ahouai (L.) A.DC. ME 692.

Tonduzia longifolia (A. DC) Markgr. JR 2464, SAY 2171.

ARALIACEAE

Dendropanax arboreus (L.) Decne. et Planch. EM 32303, FL 147, ME 1792, SAY 1959, SS 2708, 2791.

Dendropanax leptopodus (Donn.Sm.) A.C. Sm. SS 2760. 
ARISTOLOCHIACEAE

Aristolochia anguicida Jacq.

JP 12, 112, JR 2133, SAY 2935, SS 3774.

Aristolochia littoralis D.Parodi

SS 3952.

Aristolochia nelsonii Eastw.

CP 425, EM 32457, JR 1614, 1820, ME 2296, 2549, 2597,

2756, NV 496, SAY 2430, SS 2544, 2565, $2967,3141$.

\section{ASCLEPIADACEAE}

Asclepias curassavica L.

DS 95, FL 358, JR 376, 2420, ME 1707, 1987, 2884, 3449,

SAY 2448, SS 2845, 2853, 3481, 3980.

Asclepias oenotheroides Cham. et Schltdl.

ME 4401, 4475, 4548.

Blepharodon mucronatum (Schltdl.) Decne.

FL 308, JFC 697, JP 63, NV 516, 540, SAY 2519, SS 4134.

Calotropis procera (Aiton) W.T.Aiton

CP 314.

Cryptostegia grandiflora (Roxb.) R.Br.

ME 2989.

Cynanchum rensonii (Pittier) Woodson

NV 398, 569.

Dictyanthus hamatus (W.D.Stevens) W.D.Stevens

ME 3252, 3375, 3379.

Funastrum clausum (Jacq.) Schltr.

CT 476b, JFC 804, 826, JR 1918, ME 1096, 2042, 2342, 2882, SAY 2615.

Gonolobus barbatus Kunth

ME 2297.

Gonolobus chloranthus Schltdl.

JFC 690.

Gonolobus pectinatus Brandegee

JR 723, SS 2926.

Labidostelma guatemalense Schltr.

FL 172, JR 1639, ME 457.

Macroscepis diademata (Ker Gawl.) W.D.Stevens

JR 837, ME 862, 1568, 2269, 2464.

Macroscepis obovata Kunth

ME 3269, NV 361.

Marsdenia bourgaeana (Baill.) W.Rothe SS 4224.

Marsdenia callosa Juárez-Jaimes et W.D.Stevens ME 433.

Marsdenia aff. coulteri Hemsl.

ME 3222.

Marsdenia lanata (P.G.Wilson) W.D.Stevens

CP 211, JFC 754.

Marsdenia propinqua Hemsl.

CP 491, 652, ME 1445, 2076, 3571, 5086.

Marsdenia zimapanica Hemsl.

ME 3109.

Matelea cyclophylla (Standl.) Woodson

SAY 2236.

Metastelma macropoda Greenm.

NV 401, 445.

Metastelma aff. schlechtendalii Decne.

SS 3131.

Polystemma viridiflorum Decne.

ME 930, 3370, 3434.
Prosthecidiscus guatemalensis Donn.Sm. JR 1088, SAY 2472, SS 3607.

ASTERACEAE

Acmella radicans (Jacq.) R.K.Jansen

FL 352, ME 5548, 5614, 5654.

Adenophyllum aurantium (L.) Strother

JP 137, 250, ME 3978, 4282, 4428, 4560.

Ageratum corymbosum Zuccagni

JP 149, NV 435.

Ageratum microcephalum Hemsl.

FL 428, SS 3348.

Aldama dentata La Llave et Lex.

SAY 2091.

Ambrosia psilostachya DC.

EM 32122.

Aster foliaceus Lindl.

FL 258.

Baccharis salicifolia (Ruiz et Pav.) Pers.

ME 1248, 1594, 1710, 3499.

Baccharis trinervis Pers.

FL 305, JP 238, 329.

Baltimora recta $\mathrm{L}$.

NV 330.

Bidens pilosa L.

FL 212, 257, ME 1665, 3516, 4096, SAY 2029.

Bidens riparia Kunth var. refracta (Brandegee) O.E.Schulz JFC 602.

Bidens squarrosa Kunth JP 287, SS 3632, 3745.

Brickellia coulteri A.Gray var. megalodonta (Greenm.) McVaugh SAY 2843.

Brickellia diffusa (Vahl) A.Gray CP 165, 204, DS 46, JFC 545, 555, 601, JP 286, SAY 2785a, 2804a, SS 2541.

Calea integrifolia (DC.) Hemsl.

ME 5602, SAY 1993.

Calea megacephala B.L.Rob. et Greenm.

FL 265, NV 386.

Calea ternifolia Kunth.

JFC 941, JP 127, NV 419, SS 3353.

Carminatia alvarezii Rzed. et Calderón JR 1996.

Conyza apurensis Kunth

JR 2421, SAY 2090.

Conyza bonariensis (L.) Cronquist ME 1725.

Conyza canadensis (L.) Cronquist

FL 260.

Coreopsis mutica DC. var. carnosifolia Crawford NV 311, 427, SS 3427.

Cosmos sulphureus Cav. JP 23, ME 5282, SAY 2641.

Critoniopsis salicifolia (DC.) H.Rob.

MC 364, ME 1411, SS 1641, 3724, 3742, 3783.

Dahlia coccinea Cav.

SS 3071.

Decachaeta haenkeana DC. CP 731, JFC 878, JP 168. 
Decachaeta ovatifolia (DC.) R.M.King et H.Rob.

FL 435, 459.

Eclipta prostrata (L.) L.

CP 496, JFC 775, JR 336, ME 986, 1720, 2066, 5495, SS 2587.

Egletes liebmannii Sch.Bip. ex Hemsl. var. yucatana Shinners

EM 32127.

Egletes viscosa (L.) Less.

ME 125, 1848, 1902.

Elephantopus mollis Kunth

FL 445, SS 3346, 3408.

Erechtites hieraciifolius (L.) Raf. ex DC. var. cacalioides (Fisch.

ex Spreng.) Griseb.

ME 952.

Espejoa mexicana DC.

ME 767, 2293, NV 452.

Eupatorium adenophorum Spreng.

DS 55, ME 1785, 4488.

Eupatorium brevipes DC.

JP 177.

Eupatorium collinum DC.

FL 335, JP 204, SAY 2757.

Eupatorium glaberrimum DC.

JFC 947.

Eupatorium isolepis B.L.Rob.

SAY 2927.

Eupatorium cf. lozanoanum B.L.Rob. SS 2440.

Eupatorium morifolium Mill.

SAY 1950, 1984.

Eupatorium odoratum $\mathrm{L}$.

DS 92, FL 402, 415, JFC 568, JP 245, ME 1139, 2499, 2551, 3928, SAY 2771, 2792b, SS 3506.

Eupatorium pazcuarense Kunth ME 2806, 4193.

Eupatorium quadrangulare DC. DS 73, ME 1327, 1506.

Eupatorium schultzii Schnittsp.

JP 261, SAY 2736.

Eupatorium solidaginoides Kunth

CP 648, SS 3531.

Eupatorium vitifolium Klatt.

CP 808, ME 944.

Fleischmannia holwayana (B.L.Rob.) R.M.King et H.Rob. SS 2516.

Fleischmannia pycnocephala (Less.) R.M.King et H.Rob. JP 225, JR 2418, ME 4157, 4190, 4310, 5600.

Florestina latifolia (DC.) Rydb.

ME 5136.

Gnaphalium attenuatum DC. JP 195.

Gnaphalium roseum Kunth

SS 4172.

Gnaphalium semiamplexicaule DC.

FL 448.

Heliopsis aff. buphthalmoides (Jacq.) Dunal

SS 1538.

Jaumea mexicana (DC.) Benth. et Hook. f. ex Hemsl.

SS 3837.

Koanophyllon monanthum (Sch.Bip.) Ayers et B. Turner JR 2410.
Lagascea decipiens Hemsl.

JR 1009, 2000 ME 1860, 2408, 5453, SS 3605.

Lagascea helianthifolia Kunth

FL 457, JP 141, 180.

Lagascea mollis Cav.

ME 4112, 4298.

Lagascea palmeri (B.L.Rob.) B.L.Rob.

ME 3711, SS 3503.

Lasianthaea ceanothifolia (Willd.) K.M.Becker JP 179

Lasianthaea fruticosa (L.) K.M.Becker

ME 4080.

Melampodium cf. costaricense Stuessy

JR 1619.

Melampodium divaricatum (Rich.) DC.

FL 193, JFC 796, 1066, JR 454, 909, 1797, ME 1744, 2277, 2429, 3308, SS 2995, 3323.

Melampodium gracile Less.

ME 4458, 5361, 5397, 5488.

Melampodium linearilobum DC.

NV 305, 415.

Melampodium longipilum B.L.Rob.

ME 1318.

Melampodium microcephallum Less.

ME 3354.

Melampodium nutans Stuessy

CP 388, 646, JR 931, ME 2313, 800, SS 1537, 1834.

Melampodium sericeum Lag.

SAY 2651.

Melanthera nivea (L.) Small

ME 1023, 1666, 1691, 3830.

Mikania cordifolia (L.f.) Willd.

ME 4436, 4616.

Mikania micrantha Kunth

CT 454bis, DS 64, 97, ME 879,1722, 1956, 3833.

Milleria quinqueflora $\mathrm{L}$.

CP 632, JR 911, ME 2368, 3452.

Montanoa frutescens (Mairet ex DC.) Hemsl.

ME 947.

Montanoa karwinskii DC.

JFC 877.

Montanoa leucantha (Lag.) S.F.Blake subsp. arborescens (DC.)

V.A.Funk

CP 766, JP 130.

Montanoa rosei Rose ex B.L.Rob. et Greenm.

ME 902, 1068, 1174.

Montanoa tomentosa Cerv. subsp. tomentosa

JR 2001.

Montanoa tomentosa Cerv. subsp. microcephala (Sch.Bip. ex

C. Koch) V.A.Funk

JR 560, 1011, 1049, ME 1293, 2267, SS 2616.

Montanoa tomentosa Cerv. subsp. xanthiifolia (Sch.Bip. ex C.

Koch) V.A.Funk

JP 85, SAY 2824.

Neurolaena lobata (L.) Cass.

FL 462, JP 189, ME 1664, SS 3678.

Onoseris onoseroides (Kunt.) B.L.Rob.

FL 396, JP 254, JR 2162.

Otopappus epaleaceus Hemsl. SS 3448. 
Otopappus cf. imbricatus (Sch.Bip.) S.F.Blake ME 1965.

Otopappus mexicanus (Rzed.) H.Rob.

JR 1726.

Otopappus microcephalus S.F.Blake

EM 32053, JFC 724, JR 846, ME 606, 640, 780, SS 2234, 2400, 2595.

Otopappus tequilanus (A.Gray) B.L.Rob.

ME 3329.

Oxypappus scaber S.F.Blake

SS 4244.

Pectis haenkeana (DC.) Sch.Bip.

JFC 855, ME 1758, 2394, 3387, 4353, SAY 2212.

Pectis multiflosculosa (DC.) Sch.Bip.

ME 1346.

Pectis prostrata Cav.

ME 5165.

Pectis repens Brandegee

EM 32073, ME 1878.

Pectis saturejoides (Mill.) Sch.Bip. ex Seem.

ME 3170, 3986, NV 454, SS 3435.

Pectis cf. sinaloensis Fernald

ME 778.

Philactis liebmannii (Klatt) S.F.Blake

FL 267, 283.

Piqueria pilosa Kunth ME 1741.

Pityopsis graminifolia (Michx.) Nutt. var. latifolia (Fernald)

Semple et Bowers JP 328.

Pluchea odorata (L.) Cass. ME 1332, 1441, 1477, 2605, RdeS 725.

Pluchea symphytifolia (Mill.) Gillis

DS 14, ME 60, 122, 1610, 1695.

Porophyllum macrocephalum DC. CP 38, ME 764, 869, 3583, 3761.

Porophyllum punctatum (Mill.) S.F.Blake CP 203, ME 82, NV 421.

Porophyllum viridiflorum (Kunth) DC. SS 3598.

Pseudelephantopus spicatus (B.Juss. ex Aubl.) C.F. Baker ME 4078.

Pseudoconyza viscosa (Mill.) D'Arcy var. lyrata (Kunth) D'Arcy ME 839, 1658.

Pseudogynoxys chenopodioides (Kunth) Cabrera

FL 429, JP 194, 227, SAY 2760, SS 3750.

Pseudogynoxys haenkei (DC.) Cabrera

FL 413, JP 284, SS 3631.

Rumfordia floribunda DC.

JP 350.

Sabazia cf. multiradiata (Seaton) Longpre SS 1525.

Salmea scandens (L.) DC

JP 219, SAY 1954.

Schistocarpha bicolor Less.

SAY 1978.

Sclerocarpus divaricatus (Benth.) Benth. ex Hemsl.

NV 437, SS 4179, 4186.

Sclerocarpus uniserialis (Hook.) Benth. et Hook.f.

JFC 646, ME 906, 1045, NV 438, SS 3312.
Simsia lagascaeformis DC.

JP 88.

Sinclairia andrieuxii (DC.) H.Rob. et Brettell

FL 392, 433, JFC 894, JP 120, 199, SS 2524, 3514.

Sinclairia cf. caducifolia (B.L.Rob. et Bartlett) Rydb.

JP 279, 315.

Sinclairia discolor Hook. et Arn.

JP 255, ME 4069, SS 2401.

Sinclairia similis (McVaugh) H.Rob. et Brettell ME 4048.

Smallanthus mexicanus Sch.Bip.

FL 222.

Stenocephalum juncundum (Gleason) H.Rob. ME 5474.

Stevia connata Lag. JP 125.

Stevia origanoides Kunth

FL 452.

Stevia ovata Willd.

SS 3360.

Synedrella nodiflora (L.) Gaertn.

JFC 633, JP 160, SS 3523.

Tagetes erecta $\mathrm{L}$. ME 1728, SS 3480.

Tagetes filifolia Lag. SS 3412.

Tehuana calzadae Panero et Villaseñor FL 170, SS 3315.

Tithonia diversifolia (Hemsl.) A.Gray CT 487, DS 52, JP 281, ME 100, 868, 996.

Tithonia rotundifolia (Mill.) S.F.Blake CP 812, 813, FL 417, JP 146, JR 352, 2422, ME 1390, 1471, 1712, 1721, NV 479, SAY 2006, 2761, SS 3570, 3578, 3667.

Tithonia tubiformis (Jacq.) Cass.

FL 359, ME 1681.

Tridax procumbens L.

EM 32192, ME 1046, 1779, 1913, 1961, NV 354, 440, SS 1496, 3470, 3592, 3772.

Tridax tenuifolia Rose var. microcephala Rose ME 3767, 3984.

Trigonospermum melampodioides DC. CP 505, ME 3646.

Trixis inula Crantz

CT 456, EM 32099, JR 398, ME 1813, 4419.

Trixis pringlei B.L.Rob. et Greenm.

EM 32051.

Trixis pterocaulis B.L.Rob. et Greenm. JP 187, ME 5484, 5515.

Trixis silvatica B.L.Rob. et Greenm.

JFC 566, 721, ME 3983, SS 2608, 3741.

Verbesina aff. abscondita Klatt

ME 933, 988.

Verbesina crocata (Cav.) Less.

FL 395.

Verbesina myriocephala Sch.Bip. ex Klatt SS 4279, FL 401.

Verbesina oligantha B.L.Rob.

FL 420, JP 173, SAY 2758.

Verbesina persicifolia DC.

JR 877. 
Verbesina platyptera Sch.Bip. ex Klatt

SS 4221.

Verbesina turbacensis Kunth

CP 707, JP 183, ME 3808.

Vernonanthura liatroides (DC.) H.Rob

JP 289.

Vernonanthura oaxacana (Schultz-Bip. ex Klatt) H.Rob.

ME 1503.

Vernonanthura patens (Kunth) H.Rob.

SS 4431.

Viguiera cordata (Hook. et Arn.) D'Arcy

FL 453, JFC 629, ME 3741, 4151, 5358, 5473, SAY 2738, SS 3668.

Viguiera puruana Paray

SS 3687.

Viguiera tenuis A.Gray

JFC 539, 731, 1082, JP 167, ME 2448, 5382, 5415, SS 1495, 3387.

Wedelia acapulcensis Kunth

JFC 873, ME 5367, SS 1494, 1524.

Wedelia trilobata (L.) Hitchc.

FL 255, ME 3447, 3505, 4614.

Xanthium strumarium L.

ME 1846, 2036, 2487, 3715, RdeS 727.

Zinnia americana (Mill.) Olorode et A.M.Torres

JFC 746, JR 940, 1912, SS 1506.

Zinnia flavicoma (DC.) Olorode et Torres

ME 2396.

Zinnia peruviana (L.) L.

JR 929, NV 412.

Zinnia zinnioides (Kunth) Olorode et Torres

FL 108, NV 328.

\section{BASELLACEAE}

Anredera vesicaria (Lam.) C.F.Gaertn.

ME 3809.

BATACEAE

Batis maritima L.

JR 388.

\section{BEGONIACEAE}

Begonia plebeja Liebm.

JR 945.

Begonia sp. nov.

ME 948.

\section{BIGNONIACEAE}

Adenocalymna inundatum C.Mart. ex DC. ASA 54, EM 32072, 32390, FL 102, ME 1150, 1430, 2055, 2102, SS 1562, 1813, 2363.

Amphilophium paniculatum (L.) Kunth

CP 195, CT 478-A, JR 737, ME 753.

Arrabidaea floribunda (Kunth) Loes.

ME 417, 531, 755, 858.

Arrabidaea mollissima (Kunth) Bureau et K.Schum.

SS 2429.

Arrabidaea patellifera (Schltdl.) Sandwith

CP 884, JR 823, 1744, NV 338, 511.
Astianthus viminalis (Kunth) Baill.

EM 32082, JR 1200, 2256, ME 2030, 2780, 2860, 2956, SS 1786, 1980.

Crescentia cujete L.

DS 88, ME 1854, SAY 2795.

Cydista aequinoctialis (L.) Miers var. hirtella (Benth.) A.H.Gentry EM 32095.

Cydista diversifolia (Kunth) Miers CP 489, FL 336, JR 900, ME 661, 1079, 1512, 3338, SS 1903, 3036, 3392, 3581.

Cydista potosina (K.Schum. et Loes.) Loes. FL 115b, 156.

Godmania aesculifolia (Kunth) Standl. EM 32093, FL 96, JR 2334, SS 1654, 2531.

₹ Jacaranda mimosifolia D.Don ME 2151.

\# Kigelia pinnata (Jacq.) DC. SS 2805.

Macfadyena unguis-cati (L.) A.H.Gentry SS 3881.

Mansoa hymenaea (DC.) A.H.Gentry JR 2129, JR 550, ME 1574, 1705, 1763, 1885, SS 1631.

Melloa quadrivalvis (Jacq.) A.H.Gentry JP 427.

Paragonia pyramidata (Rich.) Bureau CT 458, EM 32064, JFC 888, ME 1659.

Parmentiera aculeata (Kunth) Seem. JR 400, 2202, ME 463, 1303, SS 2971, 3504.

Pithecoctenium crucigerum (L.) A.H.Gentry JR 767, ME 459, 1281, 1706, 2122, NV 262, SS 2042.

Tabebuia chrysantha (Jacq.) G.Nicholson CT 414, ET 1306, MC 372, ME 31, 1753, 2795, SS 1778, 1961, 1991, 2155, YAC 163.

Tabebuia impetiginosa (Mart. ex DC.) Standl. JR 619, ME 1941, SS 2173, 2628.

Tabebuia rosea (Bertol.) A.DC. CH 1930, DS 80, FL 79, JR 356, ME 58, 1547, 1578, 2683, RdeS 705, SS 2852.

Tecoma stans (L.) Juss. ex Kunth CP 193, DS 6, EM 32080, JP 200, ME 1058, 1242, 1294, 1466, RdeS 716, SS 3608.

Xylophragma seemannianum (Kuntze) Sandwith JR 1202, SS 2384.

BIXACEAE

‡ Bixa orellana L. var. urucurana (Willd.) Kuntze ex Pilg. DS 57.

Cochlospermum vitifolium (Willd.) Spreng. CH 1937, ET 1302, JP 249, JR 372, 606, 2149, ME 1106, 1123, 1218, 1360, SS 1585, 2620.

BOMBACACEAE

Bernoullia flammea Oliv. SS 2834.

Ceiba aesculifolia (Kunth) Britten et Baker $\mathrm{f}$. CH 1946, YAC 166.

Ceiba parvifolia Rose CP 312, ET 1293, ME 46, 1752, 1754, 1827, SS 1977.

Ceiba pentandra (L.) Gaertner MC 350, ME 1784, SS 4366. 
Pseudobombax ellipticum (Kunth) Dugand

CP 320, JR 477, MC 386, ME 69, 2681, 2794, SS 2831.

\section{BORAGINACEAE}

Bourreria purpusii Brandegee

JR 2511, ME 235, 533, SS 1866, 2360.

Bourreria rekoi Standl.

SAY 2095, SS 2756, 3873.

Cordia alliodora (Ruiz et Pav.) Oken

CH 1915, CP 207, 664, FL 390, JP 273, JR 2097, 2186, ME

2557, 5140, 5305, SAY 2233, SS 1588, 2665, 3597.

Cordia curassavica (Jacq.) Roem. et Schult.

ASA 44, DS 51, JFC 812, JR 744, 1898, ME 1641, 2164,

2221, 2235, SAY 2814, SS 1787, 2991, 3833, 3958.

Cordia dentata Poir.

EM 32176, JR 603, 1609, ME 119,1093, 1116, 1144, 1155,

RGS 415, SS 1578, 3757, YAC 132, 142.

Cordia elaeagnoides A.DC.

ET 1310, MC 399, ME 882, 1030, 1052, 1067, RGS 460, SS 1269, 1531, 4034, YAC 134.

Cordia gerascanthus L.

JR 2092, 2127, 2142, 2250, 2357, ME 2949, SAY 2044, SS $1308,3838,3846$.

Cordia globosa (Jacq.) Kunth

ME 391.

Cordia guerckeana Loes.

ET 1461, SS 1767.

Cordia inermis (Mill.) I.M.Johnst.

CP 474, EM 32405, 32448, JR 788, 826, 850, ME 487, 3249, 5232, 5238, SAY 2608, SS 1887, 3973.

Cordia oaxacana A.DC.

CP 394, CT 420, EM 32377, ME 908, 2295, 2648, SS 2944, 3390.

Cordia seleriana Fernald

ME 4324, 5887, SAY 2046, SS 3761.

Cordia sonorae Rose

ME 1655.

Cordia stellifera I.M.Johnst.

JR 2520.

Cordia stenoclada I.M.Johnst.

SS 2716, 2719.

Cordia tinifolia Willd. ex Roem. et Schult.

SAY 2020, SS 1988, 2299, 2326, 2548.

Cordia truncatifolia Bartlett

CH 1934, CP 308, CT 440, EM 3204, JR 604, 2557, ME 210,

1624, 1978, 2096, SAY 2043, SS 803, 3823, 3825, 3834.

Cordia sp. nov.

CP 96, ME 1048, 1134, SS 1629, 3590.

₹ Ehretia tinifolia L.

EM 32067, JR 366, 1775, ME 4487.

Heliotropium angiospermum Murray

DS 28, EM 32215, JR 1618, ME 2837, 2906, 3009, 3045,

SAY 2628, SS 2994.

Heliotropium curassavicum L.

ME 3268, 4650, 4708, SAY 2036.

Heliotropium fallax I.M.Johnst.

JR 941, ME 357, 375, 3760, SS 3381, 3465, 3494.

Heliotropium filiforme Lehm.

CP 90, 403, ET 1294, JFC 713, JR 386, 828, ME 400, 2239.

SS 1501, 1835, 2549.
Heliotropium indicum L.

EM 32124, JR 1218, 1631, 2553, ME 1914, 2896, 2961, 3129, SAY 2032.

Heliotropium lagoense (Warm.) Gürke ME 1028.

Heliotropium macrostachyum (DC.) Hemsl. CP 86, 519, 760, EM 32375, JR 852, 1899, ME 103, 1382, 2417, 2490, SS 1539, 1870, 1956, 2965.

Heliotropium procumbens Mill. EM 32189, ME 2337, 2582, 2814, 3501.

Heliotropium rufipilum (Benth.) I.M.Johnst. CP 739, FL 101, JP 197, ME 4210, SAY 2001, 2037, 2092, 2244, SS 2816, 3527, 3680, 3872.

Heliotropium ternatum Vahl CP 681, EM 32361, 32479, JR 2244, ME 535, 2316, SS 2656, 3831.

Tournefortia acutiflora M.Martens et Galeotti SS 3502.

Tournefortia densiflora M.Martens et Galeotti CP 11, JFC1092, JP 161, 280, JR 431, ME 1565, 1636, 1653, 2094, SAY 2452, SS 1613, 2534, 2626, 3593.

Tournefortia glabra L. EM 32171, JR 397, ME 129, 179, 1924, 1993, SS 2741.

Tournefortia hartwegiana Steud. FL 138, ME 2995, 3018, RGS 380.

Tournefortia hirsutissima L. forma bicolor (Sw) D.L.Nash ME 4630, 4429.

Tournefortia volubilis L. MC 395, ME 1891, 2776, 3216, 4813, NV 286, SS 2652.

Tournefortia sp. nov. JR 735, ME 479, 1142, 1184, 2051.

BURSERACEAE

Bursera bipinnata (Sessé et Moc. ex DC) Engl. JP 44, SAY 2572, SS 3096.

Bursera aff. cinerea Engl. CP 397, EM 32143, 32498, JP 81, ME 397, 4816, SAY 2406, SS 2341.

Bursera discolor Rzed. JP 41.

Bursera excelsa (Kunth) Engl. CP 83, EM 32371, 32389, 32402, 32404, ET 1286, 1315, JP 34, JR 433bis, 760, 1877, 2521, ME 1276, 1352, 2108, 2174, SAY 2407, 2425, 2768, SS 1809, 1867, 2242, 2436.

Bursera fagaroides (Kunth) Engl. ASA 82, EM 32383, 32664, ET 1323, JR 463, 732, 1826, ME $313,3183,3429,760$, RGS 466, SAY 2405, 2655, SS 2175 , 3224.

Bursera grandifolia (Schltdl.) Engl. EM 32372, 32392, ME 2191, SS 2438.

Bursera graveolens (Kunth) Triana et Planch. CP 412, EM 32379, 32384, 32388, 32401, JR 733, ME 293, 341, 3256, 3438, SS 2229, 2230, 2243, 2311.

Bursera heteresthes Bullock EM 32385, 32396, 32403, JR 460, 2550, ME 427, 3215, 4674, 4688, 5072, SAY 2400, 2403, 2670, SS 1573, 2144, 2309, 2335.

Bursera krusei Rzed.

CP 464, EM 32378, JR 433, 1895, ME 297, 311, 2192, 3138, SS 2160, 2387, 2402. 
Bursera laurihuertae Rzed. et Calderón

ME 2134, 3037, 3323, 4683, SAY 2417, 2662, 2676.

Bursera schlechtendalii Engl.

JR 791, 876, ME 4681, NV 369, SAY 2398, 2622.

Bursera simaruba (L.) Sarg.

ASA 66, EM 32352, 32356, 32376, 32386, FL 190, JR, 761, 1893, 1902, 2430, ME 1137, 2138, 2173, 2183, RGS 468,

SAY 1989, 2261, 2349, 2375, SS 1542, 1814, 2146, 2763.

Bursera sp. nov.

EM 32347.

Commiphora sarcopoda (P.G.Wilson) Rzed. et Palacios

SAY 2620, SS 2351.

\section{CACTACEAE}

Acanthocereus occidentalis Britton et Rose, var. nov. ME 3055.

Hylocereus undatus (Haw.) Britton et Rose

EM 32109, 32112, JR 2441, 2514, SAY 2182.

Mammillaria albilanata Backeb.

SS 2875.

Mammillaria karwinskiana Mart.

SAY 2653.

Mammillaria voburnensis Scheer var. collinsii (Britton et Rose) Repp.

SS 2567.

Neobuxbaumia tetetzo (J.M.Coult.) Backeb. var. nuda

(E.Y.Dawson) E.Y.Dawson

SS 2885.

Nopalea auberi (Pfeiff.) Salm-Dyck

SS 1969.

Nopalea dejecta (Salm-Dyck) Salm-Dyck CP 536, JFC 1095, MC 361.

Nopalea karwinskiana (Salm-Dyck) K.Schum.

ET 1468, ME 2936, 3025.

Nyctocereus oaxacensis Britton et Rose EM 32102-A, JR 2350, ME 3068, 3371.

Opuntia decumbens Salm-Dyck

ET 391, 1475, MC 396, 1518, 1519, SS 2043.

Opuntia puberula Pfeiff.

JR 2197.

Opuntia velutina F.A.C.Weber

ME 6051.

Pachycereus pecten-aboriginum (Engelm. ex S.Watson) Britton et Rose

MC 384.

Peniocereus fosterianus Cutak var. nizandensis Sánchez-

Mejorada

SS 3228.

Peniocereus oaxacensis (Britton et Rose) D.R. Hunt

JR 1828.

Pereskia lychnidiflora DC.

ASA 85, CP 68, JFC 1099, JR 380, 2543, MC 400, ME 189, 1531, 1569, 2171, SS 1865, 4093.

Pereskiopsis diguetii (F.A.C.Weber) Britton et Rose

CT 514, SS 1810.

Pereskiopsis rotundifolia (DC.) Britton et Rose

ME 2521, 6082.

Pilosocereus collinsii (Britton et Rose) Byles et G.D. Rowley

EM 32138.
Pilosocereus quadricentralis (E.Y.Dawson) Backeb. JR 2344.

Selenicereus coniflorus (Weing.) Britton et Rose SS 2910.

Stenocereus griseus (Haw.) Buxb. ME 2979.

CAMPANULACEAE

Hippobroma longiflora (L.) G.Don

CP 773, JR 1771.

Lobelia cf. berlandieri A.DC.

SS 3423.

Lobelia laxiflora Kunth

JR 2424, SS 2718.

CAPPARACEAE

Capparis admirabilis Standl.

JR 391.

Capparis baducca L.

JR 347, 2140. ME 63, 2935, SS 1859, 2675.

Capparis flexuosa $\mathrm{L}$. ASA 70, JR 713, ME 2012, 2070, 2876, 2963. SAY 2221, SS 2835, 2891, 2914.

Capparis incana Kunth JR 2249, ME 1925, 2083, 2199, 2227, SS 1310, 2172, 2364.

Capparis indica (L.) Fawc. et Rendle CH 1965, EM 32162, 32164, ET 1325, JR 884,1221, 2313, ME 166, 1826, 1929, 2074, RGS 382, 407, SAY 2062, SS 1259, 1836, 1868, 2050.

Capparis odoratissima Jacq. CH 1958, CT 472bis, ME 183, 287, 1974, 2833, RGS 392, YAC 128.

Cleome gynandra L. ME 374, 523, 554, 1998.

Cleome parvisepala Heilborn ME 2420.

Crateva tapia L. CH 1928, JR 381, 710, 1050, 2258, MC 362, ME 158, 165, 182, 1546, 1570, SS 1853, 2781, YAC 155.

Forchhammeria pallida Liebm. EM 32165, ET 1311, ME 451, 1130, 1928, 1983, SS 2061.

Morisonia americana L. CT 435, EM 32040, JR 1215, MC 376, 385, ME 230, 2098, RGS 391, 417, SAY 2050, SS 1988bis.

Polanisia viscosa (L.) DC. ASA 48, CP 5, CT 485, EM 32496, ME 114, 356, 675, 2236, SS 2618 .

CAPRIFOLIACEAE

Lonicera pilosa (Kunth) Spreng. FL 141.

Sambucus mexicana C.Presl. ex DC. FL 100, JFC 716bis.

CARICACEAE

\# Carica papaya L. JR 2504, SS 2730.

Jacaratia mexicana A.DC. CP 176, JFC 598, JR 2147, ME 1367, 1372, 1830, SS 1861. 


\section{CARYOPHYLLACEAE}

Cerastium arvense $\mathrm{L}$.

FL 38, 104.

Drymaria glandulosa C.Presl

ME 1684.

\section{CECROPIACEAE}

Cecropia obtusifolia Bertol.

DS 72, ME 25.

\section{CELASTRACEAE}

Rhacoma eucymosa (Loes. et Pittier) Standl.

JR 399, 2113.

Rhacoma uragoga (Jacq.) Baill.

ME 3106.

Schaefferia sp.

YAC 130.

\section{CHENOPODIACEAE}

Chenopodium ambrosioides L.

DS 109.

\section{CHRYSOBALANACEAE}

Chrysobalanus icaco L.

ME 5781.

Licania arborea Seem.

CH 1942, JR 1079, 2101, 2209, ME 2754, 5746, SS 2856, 4403.

\section{CISTACEAE}

Helianthemum sp.

FL 127, 131.

\section{CLETHRACEAE}

Clethra mexicana DC.

EM 32280, 32305.

\section{CLUSIACEAE}

Calophyllum brasiliense Cambess. var. rekoi (Standl.) Standl. CP 727, EM 32113, ME 1796.

Clusia salvinii Donn.Sm.

MC 357.

\section{COMBRETACEAE}

Bucida macrostachya Standl.

ASA 94, CP 18, JR 412, 448, 451, MC 378, ME 353, 1135, SS 1800.

Bucida wigginsiana Miranda

ASA 95, CH 1966, JR 616, 750, 752, 781, ME 443, SS 1633, 1885, 2984.

Combretum argenteum Bertol.

JFC 763.

Combretum fruticosum (Loefl.) Stuntz

CP 70, JP 343, 2111, MC 379, ME 1010, 1361, 1402, 1428,

SS 1603, 1775, 1981, 2433.

Combretum laxum Jacq.

JR 2152, 2212, ME 1543, 1584, 2708, 2766, SS 1594, 1788, 1982, 1986.

Conocarpus erectus L. var. sericeus Fors ex DC.

ME 286, 1629, 1717, 2959, NV 268,YAC 129.
Laguncularia racemosa (L.) C.F.Gaertn. MC 397, ME 2382, 2978, 3321, 3396, NV 265.

\section{CONVOLVULACEAE}

Aniseia cernua Moric.

ME 1083, 2620.

Evolvulus alsinoides (L.) L.

CP 6, 65, 471, 688, EM 32102, JFC 620, JR 935, ME 769, 1271.

Evolvulus cardiophyllus Schltdl.

CP 162, SS 2489, 3452.

Ipomoea alba L.

ME 2419, 2752.

Ipomoea arborescens (Humb. et Bonpl. ex Willd.) G.Don

CH 1911, EM 32103, ME 1770, 2034, 2650, 2751, SS 2350.

Ipomoea cf. batatas (L.) Lam.

EM 32078.

Ipomoea bracteata Cav.

CP 221, CT 472, JFC 807, JR 2135, MC 383bis, ME 1131, 1374, 1412, 1426, SS 1604, 2019, 2676.

Ipomoea carnea Jacq. subsp. fistulosa (Mart. ex Choisy)

D.F.Austin

SS 2569

Ipomoea dumosa (Benth.) L.O.Williams

JFC 706.

Ipomoea hederifolia L.

CP 27, CT 470, 476a, JFC 594, 677, JR 926, 1001, ME 1006, $1041,1115,1172$, SS 1517.

Ipomoea meyeri (Spreng.) G.Don

JFC 871, JR 1037, SS 2654.

Ipomoea microsepala Benth.

JFC 696, JR 2158, ME 2747, 2801.

Ipomoea minutiflora (M.Martens et Galeotti) House

EM 32671, JR 1012.

Ipomoea nil (L.) Roth

CP 444, 676, JR 912, ME 912, 2435, SS 1509.

Ipomoea pedicellaris Benth.

CP 69, 201, 689, JFC 581, 791, ME 1201, 1250, 1270, 1297 SS 1899.

Ipomoea pes-caprae (L.) R.Br.

JFC 862, MC 390, ME 1164, 1224, 1345, 1555.

Ipomoea praecana House

JFC 844, JR 843, ME 1395, 1409, 1481, SS 1277, 2447, 2674.

Ipomoea quamoclit L.

CP 445, JFC 832, SS 2607.

Ipomoea robinsonii House

EM 32348.

Ipomoea setosa Ker Gawl.

JFC 710, JR 2007, ME 1043, 1199, 1243.

Ipomoea suaveolens (M.Martens et Galeotti) Hemsl.

JR 1002, 2003.

Ipomoea ternifolia Cav. var. leptotoma (Torr.) J.A.McDonald CP 88.

Ipomoea trifida (Kunth) G.Don

CP 626, JFC 595, 616, ME 107, 1195, 1291, 2022, SS 1900. Ipomoea triloba L.

CP 197, 446, СT 448b, ME 518, 804, 2356.

Ipomoea wolcottiana Rose

ME 2779.

Jacquemontia nodiflora (Desr.) G.Don

CT 416, JFC 756. 
Jacquemontia oaxacana (Meisn.) Hallier f. SS 1493.

Jacquemontia pentantha (Jacq.) G.Don JFC 702, 703, 705.

Jacquemontia pringlei A.Gray

JFC 552, SS 2539, 2572.

Jacquemontia sphaerostigma (Cav.) Rusby JFC 672.

Merremia aegyptia (L.) Urb. CT 441, ME 928, 1027.

Merremia cissoides (Lam.) Hallier $\mathrm{f}$.

JR 1024, 2155, ME 109, 963, 1196, 2025, 2591.

Merremia quinquefolia (L.) Hallier $\mathrm{f}$.

ME 2630, 2914.

Merremia umbellata (L.) Hallier $\mathrm{f}$.

JR 2208, MC 389, ME 1200, 1203, 1595, 1714.

Operculina pteripes (G.Don) O'Donell

CP 645, JR 1019, SS 1902.

Turbina corymbosa (L.) Raf.

JFC 801, ME 987, 1192, 1254.

\section{CRASSULACEAE}

Echeveria sp.

JR 348.

\section{CUCURBITACEAE}

Cayaponia attenuata (Hook. et Arn.) Cogn.

JR 2206, ME 1225, 1296, 1485, 2013.

Cayaponia racemosa (Mill.) Cogn.

CT 470, JFC 627, ME 2206, SS 2494.

Chalema synanthera Dieterle

ME 681, SS 1516, 1566.

Cucumis anguria L.

CP 89, JFC 1089, JR 1087, ME 841, 1532, RGS 390.

Cucurbita argyrosperma C.Huber subsp. sororia (L.H.Bailey)

Merrick et D.M.Bates CP 91, 669, CT 469, ME 718, 806, SS 1901.

Cucurbita pepo L.

ME 2335.

Cyclanthera multifoliola Cogn.

CP 79, ME 816, 880, 895, 901.

Echinopepon paniculatus (Cogn.) Dieterle

JFC 662, ME 798, 807, 851, 872, SS 1535, 1561.

Echinopepon racemosus (Steud.) C.Jeffrey

CP 78, 662, 699, ME 935.

Ibervillea hypoleuca (Standl.) C.Jeffrey

CT 412, 522, JR 465, ME 416, 551, 648, SS 1877, 2238, 2247, 2394, YAC 133.

Luffa cylindrica M.Roem.

CT 477, ME 1065, 1236, 1461, 2413, 2712.

Melothria pendula L.

CP 72, 429, 528, 660, CT 519, JR 2419, ME 1331, 1596, 1819, 1875.

Momordica charantia L.

CT 475bis, DS 41, JR 746, ME 1025, 1097, 1328, 2006, SS 1792, 2591.

Polyclathra albiflora (Cogn.) C.Jeffrey

ME 1047, 1415.

Polyclathra cucumerina Bertol.

CP 672, JR 2002, ME 2334.
Rytidostylis gracilis Hook. et Arn. CP 8, 451, 643, 738, CT 479, 481, EM 32663, JFC 574, 617, 760, 798, JR 918, ME 909, 1008, 2380.

Schizocarpum palmeri Cogn. et Rose CP 28, ME 734, 849, 873, SS 1563.

Sechiopsis triqueter (Ser.) Naudin CP 695.

Sicydium schiedeanum Schltdl. CP 401, JR 1816, ME 2351.

Sicyos deppei G.Don

CP 671, JR 2013, ME 1021, SS 2510.

DILLENIACEAE

Curatella americana L. JR 601, SS 4438.

EBENACEAE

Diospyros aequoris Standl. ME 2980.

Diospyros digyna Jacq. JR 1763.

Diospyros salicifolia Humb. et Bonpl. ex Willd. CT 462a, JR 466, 895, 2122, ME 1075, 1212, 1351, 1630, SS 1291, 2047, 3213.

ERYTHROXYLACEAE

Erythroxylum havanense Jacq. ME 349.

EUPHORBIACEAE

Acalypha alopecuroidea Jacq. ME 499, 813.

Acalypha arvensis Poepp. et Endl. ME 1740.

Acalypha setosa A.Rich. SS 1519

Acalypha subviscida S.Watson SS 1520.

Alchornea latifolia Sw. SS 2768.

Chamaesyce densiflora (Klotzsch et Garcke) Millsp. JR 339.

Chamaesyce dioica (Kunth) Millsp. ASA 60, CP 84, 391, EM 32485, JR 804, SS 1831.

Chamaesyce hirta (L.) Millsp. CT 480bis, DS 48, EM 32469, JFC 652, 768, 908, JR 936, ME 2264, 2301, 4537.

Chamaesyce hyssopifolia (L.) Small JFC 904, ME 2142, 2665.

Chamaesyce lasiocarpa (Klotzsch) Arthur ASA 59, JFC 573, 743, JR 805.

Chamaesyce aff. serpens (Kunth) Small EM 32125.

Chamaesyce thymifolia (L.) Millsp.

JFC 858, JR 1212, ME 852.

Cnidoscolus tubulosus (Müll.Arg.) I.M.Johnst. ET 1324.

Croton alamosanus Rose ET 1465, SS 2060, 2156, 2546. 
Croton axillaris Müll.Arg.

SS 1948.

Croton draco Schltdl.

SAY 2266.

Croton fragilis Kunth

ASA 55, JR 776, ME 350, 405, 482, 536, SS 1820, 1837, 2228, 2339.

Croton guatemalensis Lotsy

ET 1303, SS 1247.

Croton pseudoniveus Lundell

JR 1728.

Croton ramillatus Croizat

ASA 87, JR 736, 783, ME 440.

Croton reflexifolius Kunth

SS 1558.

Croton septemnervius McVaugh

JFC 1102, SS 2142, 2325, 2468.

Croton suberosus Kunth

ASA 76, CT 417, ET 1314, JR 422, 730, 743, 779, MC 375,

ME 342, 454, 485, 547, SS 1821.

Croton xalapensis Kunth

SAY 2070.

Dalechampia scandens L.

CP 410, 460, EM 32454, JFC 752, 811, JR 1821, ME 490, 685, 2249, 775, SS 1534.

Dalembertia triangularis Müll.Arg.

CP 517.

Ditaxis guatemalensis (Müll.Arg.) Pax et K.Hoffm.

SS 3496.

Euphorbia calyculata Kunth

EM 32052, 32446, JR 2303, SS 1838.

Euphorbia colletioides Benth.

ET 1469, ME 2661, SS 2028, 2235, 2367, 2661.

Euphorbia dioscoreoides Boiss.

CP 52, 647, 718, JR 1814, 933, ME 2554, SS 2488.

Euphorbia francoana Boiss.

CP 74, JR 930.

Euphorbia graminea Jacq.

CP 791, EM 32178, JFC 619, 626, JR 1786, ME 2370, 2669.

Euphorbia heterophylla L.

CP 435, EM 32193, ME 1026, 1745, 2003, 2425.

Euphorbia hypericifolia L.

CP 419, 431, 433, 508, CT 488, EM 32181, JR 1590, ME 2002.

Euphorbia oaxacana B.L.Rob. et Greenm.

CP 516, EM 32487, JFC 548.

Euphorbia ocymoidea L.

CP 59, JFC 684.

Euphorbia pulcherrima Willd. ex Klotzsch

DS 53, JP 262, 334.

Euphorbia schlechtendalii Boiss.

JR 2090, ME 1124.

Euphorbia segoviensis (Klotzsch et Garcke) Boiss.

CP 423, CT 475, JR 2006, SS 2600.

Garcia nutans Vahl

JFC 874, JR 1587, 1813, 2343.

Hura polyandra Baill.

CH 1944, JFC 967, JR 556, 1046.

Jatropha curcas L.

ME 315.
Jatropha gossypifolia L.

EM 32134.

Jatropha malacophylla Standl.

ME 32365, 32381, JR 792, 842, 855, ME 565, 736, SS 2154.

Jatropha sympetala S.F.Blake et Standl.

CP 22, EM 32321, ME 2119, 2175, 2224, 2326, SS 1553, 1763, 4446, 4447.

Manihot oaxacana D.J.Rogers et Appan

JR 421, 438, 443, 475.

Margaritaria nobilis L.f.

ASA 79, JR 1640, 1891.

Omphalea sp.

JR 385.

Phyllanthus elsiae Urb.

ME 3914.

‡ Ricinus communis $\mathrm{L}$.

DS 40, ME 11, 12, 13, 1544.

Sapium macrocarpum Müll.Arg.

SS 1818.

Sebastiania pavoniana (Müll.Arg.) Müll.Arg.

CP 21, ME 404, 541, SS 1815, 1882, YAC 159.

Tragia glanduligera Pax et K.Hoffm.

JFC 753.

FAGACEAE

Quercus conspersa Benth. SS 2509, 2527, 2841.

Quercus crispifolia Trel.

FL 95.

Quercus glaucescens Humb. et Bonpl. SS 2508.

Quercus magnoliifolia Née SS 2840, 2842.

Quercus peduncularis Née CP 809, SS 2491.

Quercus aff. planipocula Trel. SS 2752, 2770, 2776.

Quercus segoviensis Liebm. FL 32.

\section{FLACOURTIACEAE}

Casearia arguta Kunth CP 798, SS 2533.

Casearia nitida (L.) Jacq. ASA 67, 88, CT 451, 463a, EM 32048, 32077, 32152, 32369, ET 1273, 1317, JR 390, 414, 2329, 2351, ME 188, 2017, 2052, 2095, SS 1262, 1287, 2357.

Casearia obovata Schltdl.

JR 1792.

Casearia tremula (Griseb.) Griseb. ex C. Wright ASA 57, EM 32394, JR 725, 771, ME 2176, SS 1894.

Homalium trichostemon S.F.Blake JP 380, JR 358, 471, 1613, 2151, ME 472, 754, 1786, SS $1593,4418$.

Muntingia calabura L.

JP 310, JR 715, MC 387, ME 1404, 1609, 1910, 2016.

Xylosma velutinum (Tul.) Triana et Karst.

EM 32302, 32304. 
GESNERIACEAE

Achimenes flava C.V.Morton

SS 3331.

Achimenes obscura C.V.Morton

CP 748, 768, JR 1773.

Moussonia deppeana (Schltdl. et Cham.) Hanst. SS 2742 .

\section{HERNANDIACEAE}

Gyrocarpus americanus Jacq.

JFC 739, JR 1053, 1915, 2012, 2194, ME 1413, SS 1569, 2670.

Gyrocarpus mocinnoi Espejo

ET 1312, JR 425, 435, 455, 2253, ME 1056, 1127, 1366, 2654, SS 2562.

\section{HIPPOCRATEACEAE}

Hippocratea celastroides Kunth

CP 9, EM 32120, JR 2093, 2108, 2228, ME 161, 1822, 2047, 2100, RGS 420, SS 1306, 1610, 1790.

Hippocratea excelsa Kunth CT 463b, EM 32126, 32296, JR 1201, 1208, ME 1800, 2058, 2062, 2469, RGS 400, SS 2428.

Hippocratea volubilis L. JFC 949.

\section{HYDROPHYLLACEAE}

Wigandia urens (Ruiz et Pav.) Kunth

FL 443, JP 307, 373, JR 2128, ME 1138, 1182, 1955, 2053, SS 1605.

\section{JULIANACEAE}

Amphipterygium adstringens (Schltdl.) Standl. CP 24, CT 423, JR 427, 449, 809, ME 283, 1133, SAY 2323, SS 1543, 1840, 3230, 3231.

\section{KRAMERIACEAE}

Krameria cuspidata C.Presl

DS 59, EM 32301, JFC 914.

\section{LAMIACEAE}

Hyptis capitata Jacq.

JFC 625, 771, ME 1253, 1718, 2426, 3743, NV 547, SS 3362.

Hyptis mociniana Benth.

CP 208, JR 2248, ME 1323, 1600, RdeS 728.

Hyptis mutabilis (Rich.) Briq.

JFC 683, ME 1699.

Hyptis pectinata (L.) Poit.

JFC 755, ME 1579, 1870, SS 2602.

Hyptis perpulcher Epling

CP712.

Hyptis rhomboidea Mart. et Galeotti

JFC 939, ME 810, 2493.

Hyptis suaveolens (L.) Poit.

CP 45, 411, 696, JR 1936, ME 784, 1657, 2355, 2421, SS

1505, 2525.

Hyptis tomentosa Poit.

JP 355, JR 2230, ME 1876, 1951, 1970, 2049, SS 2680.

Ocimum micranthum Kunth

JFC 675, JR 1735, 1811.
Salvia aff. fluviatilis Fernald

ME 2146.

Salvia herbacea Benth.

CP 399, JR 798, ME 553, 680, 790.

Salvia lasiocephala Hook. et Arn.

CP 724, 799.

Salvia misella Kunth

CP 164, 473, 678, DS 93, JFC 613, 615, JR 2247, ME 795, 1780, 1874, 2730, SS 2554, 2655.

Scutellaria drummondii Benth.

FL 132, SS 3712.

LEGUMINOSAE

M. en C. Mario Sousa Sánchez

Acacia acatlensis Benth. ME 247, 386, 1055, 5877, SS 2007.

Acacia cochliacantha Humb. et Bonpl. ex Willd. ASA 71, JFC 864, JR 741, 784, 2522, ME 228, 1476, 2163, 2217, SAY 2435, SS 2612, 3495.

Acacia collinsii Saff.

CH 1950, ME 1080, 2202, 2402, 2928, SS 2317, 2596.

Acacia cornigera (L.) Willd. ME 62,142, 1942, 2690.

Acacia coulteri Benth. ME 1031, 1170, 1935, 2088.

Acacia farnesiana (L.) Willd. CH 1951, EM 32131, JR 2554, ME 1626, 1755, 2208, 2532, SAY 2038.

Acacia hayesii Benth. ME 951.

Acacia hindsii Benth. CH 1910, JFC 766, JP 229, ME 1400, 1708, 1919, 2028.

Acacia macracantha Humb. et Bonpl. ex Willd. JFC 1096, ME 855, 2750, 3757.

Acacia pennatula (Cham. et Schltdl.) Benth. SAY 2592, SS 2868, 3137, 3704.

Acacia picachensis Brandegee ASA 45, JR 745, 769, ME 239, 252, 2127, 2403, SS 1841, 1884.

Acacia polyphylla DC. CP 765, 793, FL 422, JFC 885, SS 3865.

Acacia schaffneri (S.Watson) F.J.Herm. ME 1358.

Acacia tenuifolia (L.) Willd. JFC 806, JR 1076, 1635, 2518, NV 345.

Acaciella angustissima (Mill.) Britton et Rose FL 389, JP 46, JR 1901, ME 724, 774, 3478, NV 478, SAY 2468, 2501, 2557, SS 1528, 2585, 3329.

Acaciella villosa (Sw.) Britton et Rose SS 2241, ME 3440, NV 439, 576, SS 4129.

₹ Acrocarpus fraxinifolius Wight et Arn. SS 2782.

Adenopodia oaxacana M.Sousa JFC 1090, JR 2163, ME 1583, 1618, 2092, 2697, SS 3776. Aeschynomene americana L. CP 801, 815, FL 446, JFC 780, 842, 896, ME 2887, 3659, 3709, 3774, SS 2499, 3413, SS 3488.

Aeschynomene brasiliana (Poir.) DC. SAY 2442. 
Aeschynomene compacta Rose

EM 32382.

Aeschynomene fascicularis Schltdl. et Cham.

JR 854, ME 683.

Aeschynomene pinetorum Brandegee

JFC 957.

Albizia adinocephala (Donn.Sm.) Britton et Rose

CH 1913, ME 1756, 1761, 5403.

Albizia niopoides (Benth.) Burkart

JR 365, 666, 2245, NV 325, SS 2827, 2858.

Albizia occidentalis Brandegee

EM 32172, JR 1048, 2306, 2349, ME 1036, 2861, 2950,

2999, RGS 379, SAY 2060, SS 1771, 2240, 2393, 2862, ME $3331,3430,3476$.

Andira inermis (W.Wright) Kunth ex DC.

CP 318, EM 32088, JR 468, 1203, 1602, ME 1887, 2040,

2771, 2788, RdeS 702, 721, SS 2068, 2515, 2855, 3103.

Apoplanesia paniculata C.Presl

CT 435bis, ET 1277, JR 1994, ME 1070, 1112, 1121, 1556,

SS 1546, 1577, 3538, 3561, YAC 127.

Bauhinia cookii Rose

SAY 2563.

Bauhinia divaricata $\mathrm{L}$.

$\mathrm{CH} 1917, \mathrm{CT} 473 \mathrm{bis}, \mathrm{JFC} 738$, JP 110, 267, 305, 397, JR 384, 392, 2099, 2139, ME 1183, 1378, 1403, 1419, SAY 2049, SS 1279, 1527, 1635, 2013.

Bauhinia subrotundifolia Cav.

JR 2234, ME 145, 3997, 5507.

Bauhinia ungulata L.

CP 219, EM 32398, FL 133, JFC 764, JP 299, JR 2160, ME

77, SAY 2841, SS 3628, 3699, 3737.

Brongniartia bracteolata Micheli

JR 1745, 2323, 2529, ME 2144, 3069, 3343, 3406, SAY 2316, SS 2386, 2978, 3692.

Caesalpinia cacalaco Bonpl.

ME 2655.

Caesalpinia coccinea G.P.Lewis et J.L.Contr.

CH 1953, JR 622, ME 1215, 3964, 3994, 4517, SS 1951.

Caesalpinia coriaria (Jacq.) Willd.

CH 1933, JR 2106, ME 1105, 1216, 2482, 2701, SS 3461,

3967, YAC 168.

Caesalpinia eriostachys Benth.

CH 1935, CP 31, 311, EM 32154, ET 1316, JR 605, MC 369, ME 1126, 1376, 1414, 1418, SAY 2047, SS 1265, 1292, 1965, 2559.

Caesalpinia exostemma DC.

DS 54, SS 2860.

Caesalpinia platyloba S.Watson

CP 23, SS 1554, 2361.

₹ Caesalpinia pulcherrima (L.) Sw.

CT 516, DS 56, JFC 813, JP 156, ME 14, 1438, 1472, 1492,

SAY 2791, SS 2993, 3060, 3721, 4020.

Caesalpinia sclerocarpa Standl.

CP 17, JFC 540, JR 378, 839, 869, ME 1057, 1108, 2090,

3217, SS 2484.

Caesalpinia velutina (Britton et Rose) Standl.

ET 1320, 1474, JR 797, ME 249, 288, 1633, 2212, RGS 405,

SAY 2415, SS 1261, 1290, 1891, 2237, YAC 150.

‡ Cajanus cajan (L.) Millsp.

JFC 534.
Calliandra calothyrsus Meisn.

FL 184, 328, JR 1633, 1638, ME 595, 2148.

Calliandra emarginata (Humb. et Bonpl. ex Willd.) Benth.

ME 4983, SAY 2659, SS 3968, 4128.

Calliandra houstoniana (Mill.) Standl.

SS 2455.

Calliandra juzepczukii Standl.

ME 1794, SAY 2354.

Calliandra tergemina (L.) Benth.

ME 3161, 3639.

Calopogonium caeruleum (Benth.) C. Wright ex Sauvalle ME 1255, 1325, 1392, 1442, SS 3613.

Calopogonium mucunoides Desv. CP 698, JFC 915, JR 2243, ME 2633, 3769, 3958, 4020.

Canavalia acuminata Rose CT 485bis, 600 JFC 866, JR 2098, ME 1575, SAY 2816, SS 2574.

Canavalia brasiliensis M.Martens ex Benth. CT 471bis, JFC 865, JP 153, ME 1191, 1251, 1597, 1662.

Canavalia palmeri (Piper) Standl. NV 380.

Canavalia rosea (Sw.) DC.

ME 1483, 3670, 4502, 4521, NV 287, 360.

Canavalia villosa Benth.

SS 2506.

Centrosema fuscus Benth.

JP 174.

Centrosema macrocarpum Benth.

JR 2169, ME 2608, SAY 2071, SS 3695, 3748.

Centrosema molle Mart. ex Benth.

JFC 881, ME 1197, 2584, 2924, 3861, SS 2490.

Centrosema plumieri (Turpin ex Pers.) Benth.

FL 419, JR 2207, ME 1190, 1259, 1399, 1605, SAY 2743.

Centrosema sagittatum (Humb. et Bonpl. ex Willd.) Brandegee

ex L. Riley

JFC 1075.

Chaetocalyx brasiliensis (Vogel) Benth.

ME 4349.

Chaetocalyx scandens (L.) Urb.

CP 202, EM 32553, JFC 1043.

Chamaecrista flexuosa (L.) Greene

NV 302, 418, 433, SAY 2363, SS 3419.

Chamaecrista hispidula (Vahl) H.S.Irwin et Barneby

EM 32317, JFC 854, JP 322, ME 2372, 2982, 3627, 4884, NV 417, SAY 2427, 2767.

Chamaecrista nictitans (L.) Moench var. jaliscensis (Greenm.)

H.S.Irwin et Barneby CP 440, CP 675, EM 32657, JFC 576, 659, ME 783,1003, 3686.

Chamaecrista rotundifolia (Pers.) Greene

NV 503, 545, 546, SS 3365, 3406, 4159.

Chloroleucon mangense (Jacq.) Britton et Rose CP 34, JR 711, ME 1050, 1143, 2135, 2445, SS 1785, 2603, 3458.

Clitoria falcata Lam.

JFC 934, 960.

Clitoria ternatea L.

ME 976, 2411, 4574.

Cojoba arborea (L.) Britton et Rose

JR 2507, SAY 2017, SS 2814. 
Coursetia caribaea (Jacq.) Lavin var. pacifica (M.Sousa et Lavin) Lavin

SAY 2314, 2809, SS 2897, 3730, 3764.

Coursetia caribaea (Jacq.) Lavin var. trifoliolata (Rydb.) Lavin JR 2310, 2354.

Coursetia glandulosa A.Gray

CH 1938, JR 2125, ME 2770, 2933, 2955, 4114, SS 2460, 2640.

Coursetia mollis B.L.Rob. et Greenm.

ME 2953, SS 3717, 3820.

Crotalaria acapulcensis Hook. et Arn.

SS 4146.

Crotalaria cajanifolia Kunth

CP 823, JFC 678, JR 910, ME 707, 3423, 3679, 3807, 5283, NV 392.

Crotalaria incana L.

ME 3621, 3956, 4229, 4807.

Crotalaria longirostrata Hook. et Arn.

JP 4, ME 3629, 3782, 4195.

Crotalaria mollicula Kunth

ME 1589, 1698, 1950.

Crotalaria pumila Ortega

CP 205, 627, JFC 1081, 782, JR 1007, 1887, 948, ME 3671, 3688, 3810, 3894, NV 497, SS 4056.

Crotalaria sagittalis $\mathrm{L}$.

NV 539, SAY 2510, 2578, SS 3073, 4171.

Dalbergia congestiflora Pittier

JFC 1087, JR 2233, ME 2660.

Dalbergia glabra (Mill.) Standl.

JFC 695, ME 2157, 3004, SS 4135.

Dalbergia granadillo Pittier

CP 313, EM 32057, JFC 937, JR 1074, 2510, ME 467, 1292, 2947, SAY 2579, SS 2890, 3948.

Dalea sp.

ME 2546, 4072, NV 390, SS 2526, 2537, 3420.

Desmanthus virgatus (L.) Willd.

ME 3276, 3922, 4841, 4995.

Desmodium adscendens (Sw.) DC.

SAY 2491.

Desmodium barbatum (L.) Benth.

CP 701, JFC 961, ME 3657, 3674, 3859, NV 375, 402, 430, 508, SS 3355, 3426, 4163.

Desmodium cajanifolium (Kunth) DC.

EM 32349.

Desmodium distortum (Aubl.) J.F.Macbr.

JFC 636, ME 3940, 4105.

Desmodium glabrum (Mill.) DC.

ME 950, SAY 2790, SS 3542.

Desmodium hookerianum D.Dietr.

ME 3829, SS 3483.

Desmodium incanum DC.

CP 771, JFC 645, 670, 895, 943, JR 1804, SAY 2334, 2361, 2529, 2533, SS 2819, 3090, 3127, 3347.

Desmodium infractum DC.

CP 804, JP 67, NV 376, 387, 483, SAY 2631, 2634, SS 2492, $3345,4150$.

Desmodium intortum (Mill.) Urb.

JP 210.

Desmodium procumbens (Mill.) Hitchc.

JFC 611, 836.
Desmodium scorpiurus (Sw.) Desv.

EM 32190, JFC 726, ME 2900, 2905, 2919, 3944, SS 2563, $3418,3617,3759$.

Desmodium tortuosum (Sw.) DC.

JFC 810.

Desmodium triflorum (L.) DC.

NV 447.

Diphysa americana (Mill.) M.Sousa

JP 106, SS 3700, 4374.

Diphysa echinata Rose ME 4809.

Diphysa floribunda Peyr. SS 4375.

Diphysa humilis Oerst. ex Benth et Oerst. JP 321, JR 2347, ME 2564, 4717, SS 2638.

Diphysa aff. puberulenta Rydb. JR 2200.

Diphysa suberosa S.Watson SAY 2364.

Diphysa aff. thurberi (A.Gray) Rydb. ex Standl. JFC 823.

Entadopsis polystachia (L.) Britton CH 1957, CP 93, FL 330, ME 421, 1217, 3288, 3911, SAY 2215, SS 1589, 1772, 3707.

Enterolobium cyclocarpum (Jacq.) Griseb.

CH 1945, MC 402, ME 1439, 1490, 1608, 1890, RdeS 706, SS 2009, 4430.

Eriosema crinitum (Kunth) G.Don

JFC 959, SS 2501, 3101.

Eriosema diffusum (Kunth) G.Don

JFC 917, NV 373, SAY 2597.

Eriosema grandiflorum (Schltdl. et Cham.) G.Don JP 186, SAY 2765.

Erythrina lanata Rose CP 217, JR 812, ME 1362, 1373, 1502, 1527, SS 1766, 1911, 2041, 2639.

Galactia latisiliqua Desv. ME 959.

Galactia spiciformis Torr. et A.Gray ME 3772.

Galactia striata (Jacq.) Urb. var. acapulcensis (Jacq.) Urb. JFC 840.

Gliricidia sepium (Jacq.) Kunth ex Walp. CP 222, DS 47, JR 450, 2333, ME 1408, 1437, 1535, 1585, RdeS 722, SAY 1965.

Haematoxylum brasiletto H.Karst. $\mathrm{CH}$ 1947, JR 739, 2238, RGS 410.

Havardia campylacanthus (L.Rico et M.Sousa) Barneby et J.W.Grimes CH 1936, ME 152, 1824, 1930, 2082, SAY 2019, SS 1801, 2296.

Hybosema ehrenbergii (Schltdl.) Harms ME 2129.

Hymenaea courbaril L. DS 75, JR 598, NV 294, RGS 413, SS 4404.

Indigofera hirsuta L. ME 5123, 5559.

Indigofera jamaicensis Spreng. CP 800, EM 32374, JFC 781, JR 1089, ME 2553, 3108, 3230, 3439, NV 485, 512, SS 2511, 4144. 
Indigofera lancifolia Rydb.

FL 129, JR 2499, ME 2941, 3612, 4534, 5103, NV 348.

Indigofera lespedezioides Kunth

NV 374, 543, SAY 2594, SS 2502, 2876, 3014, 3087.

Indigofera micheliana Rose

NV 343.

Indigofera microcarpa Desv.

ME 2537, 2960, 3892, 4515.

Indigofera miniata Ortega

ME 3350, 3713, 3770, 3943, SS 4028.

Indigofera platycarpa Rose

ME 2377, 3291, 3594, 3638, SAY 2770, SS 2953.

Indigofera salmoniflora Rose

CP 12, ME 922.

Indigofera suffruticosa Mill.

DS 100, ME 1229, 2029, 2346, 2815, SAY 2588.

Indigofera thibaudiana DC.

JFC 663, ME 3426, SS 3384.

Inga eriocarpa Benth.

SAY 1996.

Inga paterno Harms

EM 32345, FL 188, JR 2489, SAY 2012, SS 2761, 3645.

Inga pavoniana G.Don

SS 3851.

Inga punctata Willd.

CP 746, FL 31, 373, SAY 2155, 2187, SS 2514, 2720, 3893.

Inga vera Willd.

DS 90, EM 32283, FL 18, JR 355, 860, ME 1582, 1793, 1888, 2009, SAY 2376, SS 2044, 2795, 3620, 4415.

Leucaena esculenta (Moc. et Sessé ex A.DC.) Benth. ME 3040.

Leucaena lanceolata S.Watson var. lanceolata

ME 3771, SS 3456.

Leucaena lanceolata S.Watson var. sousae (S.Zárate) C.E.Hughes ME 3694.

Leucaena leucocephala (Lam.) de Wit subsp. glabrata (Rose)

S.Zárate

ME 1421, 4621, 4685.

Leucaena macrophylla Benth. subsp. istmensis C.E.Hughes SAY 2087.

Lonchocarpus constrictus Pittier

CH 1941, EM 32497, JR 418, 617, 731, 1878, ME 1034, 1169, 1510, 2081, SS 1298, 2150, 2244, 2298.

Lonchocarpus emarginatus Pittier

CH 1939, CP 414, ET 1470, JFC 547, JR 445, 2123, 2146 , ME 1101, 2725, 2767, 5408, SAY 2675, SS 1305, 1628, 1761, 2322.

Lonchocarpus eriocarinalis Micheli CP 520.

Lonchocarpus guatemalensis Benth.

FL 99, JR 341, 387.

Lonchocarpus hermannii M.Sousa

EM 32049, 32056, 32137, JR 2311, 2358, ME 223, 1644 , 3766, 3891, SAY 2022, SS 1776, 3841.

Lonchocarpus hintonii Sandwith SAY 1966, SS 3337, 3932.

Lonchocarpus lanceolatus Benth.

ASA 41, 80, EM 32139, JR ,2239, ME 229, 1005, 2209, 2248,

SAY 2325.

Lonchocarpus lineatus Pittier

SAY 2835, 2848, SS 3518, 4381.
Lonchocarpus longipedicellatus Pittier ASA 96, ME 910.

Lonchocarpus molinae Standl. et L.O.Williams

JR 2312, SAY 2200, 2206, SS 3301, 3343.

Lonchocarpus phaseolifolius Benth. CP16, EM 32170, 32502, 32503, JR 1023, 773, 952, ME $656,2547,3163,3468$, NV 326, 335, RGS 465, SS 2030 , 3500, 3573, 3770.

Lonchocarpus rugosus Benth. NV 370, SS 3006.

Lonchocarpus schiedeanus (Schltdl.) Harms JR 880.

Lonchocarpus sp. nov. 1 SAY 2869, 2913, SS 4377.

Lonchocarpus sp. nov. 2 CP 513, EM 32491.

Lysiloma acapulcense (Kunth) Benth. NV 298.

Lysiloma divaricatum (Jacq.) J.F.Macbr.

ME 3111, SS 2570.

Lysiloma microphyllum Benth.

ASA 62, CT 471, ET 1322, FL 332, 391, JR 740, 756, 801, 1626, ME 330, 451, 4142, 4763, SAY 2764, SS 1571, 1842, 2916, 2987.

Machaerium acuminatum Kunth SS 2826.

Machaerium biovulatum Micheli SS 3508 .

Machaerium salvadorense (Donn.Sm.) Rudd ME 1272, 1539, 2742, 4427, SS 3747.

Macroptilium atropurpureum (Seseé et Moc. ex DC.) Urb. CP 494, JFC 624, JP 266, 311, JR 2335, ME 3987, 5425, 5487, SAY 2416, 2428, 2589, SS 2611.

Macroptilium longipedunculatum (Mart. ex Benth.) Urb. JR 1005, ME 3785, SS 3382, 3466, 3535.

Marina sp.

SS 2503

Microlobius foetidus (Jacq.) M.Sousa et G. Andrade JR 395, SS 2976, YAC 149, EM 32408.

Mimosa acantholoba (Humb. et Bonpl. ex Willd.) Poir. var. acantholoba

JFC 802, 835, ME 856, 3665, 3837, NV 461, SS 3560.

Mimosa acantholoba (Humb. et Bonpl. ex Willd.) Poir. var. eurycarpa (B.L.Rob.) Barneby

CT 434, JR 790, ME 924, 3364, 2397, SS 2960.

Mimosa affinis B.L.Rob.

JFC 609, JR 944, ME 3938, NV 344, 529.

Mimosa albida Humb. et Bonpl. ex Willd. var. glabrior B.L.Rob. JR 920, ME 3559, 3611, 3630, 3787, NV 300.

Mimosa albida Humb. et Bonpl. ex Willd. var. pochutlensis

R.Grether

ME 2215, 2561, CP 71, FL 119, JFC 750, JR 1637, NV 460, 470, SS 1856, 2815.

Mimosa antioquensis Killip ex Rudd var. isthmensis R.Grether FL 217, 384, JFC 809, JR 1018, ME 3557, 3624, 3789, 5479, SS 3701.

Mimosa arenosa (Willd.) Poir.

JFC 863, ME 274, 1128, SS 1551.

Mimosa candollei R.Grether

JR 1886. 
Mimosa pigra L. var. berlandieri (A.Gray ex Torr.) B.L.Turner JR 714, ME 1231, 1342, 1856, 2027.

Mimosa pigra L. var. pigra

ME 1162, 2892, 3908, 4227.

Mimosa platycarpa Benth.

ME 1077.

Mimosa psilocarpa B.L.Rob.

JFC 593, JP 319, JR 2482, SS 3328, 4044.

Mimosa pudica L.

SS 4185.

Mimosa quadrivalvis L. var. distachya (Moc. et Sessé ex DC.)

Barneby

FC 644.

Mucuna sloanei Fawc. et Rendl.

CP 816, ME 1972, 2457, 2611, 2773.

Myrospermum frutescens Jacq.

ET 1304, JR 608, 2198, 2257, ME 245, SS 2336, 2375.

Neptunia plena (L.) Benth.

ME 1723, 3529, 3701, 3907, SS 3199.

Nissolia fruticosa Jacq.

JR 899, 1903, ME 643, 691, 3435, 5251, NV 359, SS 3453, 3580, 3987, 4038.

Nissolia microptera Poir.

JR 1968.

Pachyrhizus erosus (L.) Urb.

JFC 664.

Pachyrhizus ferrugineus (Piper) M.Sorensen

NV 541.

Pachyrhizus vernalis R.T.Clausen

FL 263, NV 383, SS 3013.

Phaseolus leptostachyus Benth.

JFC 680.

Phaseolus lunatus L.

ME 1484, 4009, 4158, 4176, RdeS 726, SS 3762.

Phaseolus micranthus Hook. et Arn.

JP 87, SAY 2621, SS 4070.

Phaseolus microcarpus Mart.

CP 640, ME 3618, NV 573, SS 3388, 3541.

Piptadenia flava (Spreng. ex DC.) Benth.

CP 13, ME 3582, 3591, 3697, 4060, SS 2345, 3540, 3558, 4095.

Piptadenia obliqua (Pers.) J.F.Macbr.

CH 1912, CP 87, CT 512, EM 32191, 32400, JR 2126, 2304, 2353, 446, MC 371, ME 1002, 1039, 1175, 1383, SS 1303, 2008, 2918, 3583, YAC 145.

Piscidia carthagenensis Jacq.

JR 389, 716, 2252, 2254, ME 1966, 2045, 2755, 2851, SAY

2054, SS 1253, 1268, 1616, 1984.

Pithecellobium dulce (Roxb.) Benth.

DS 50, 99, JR 1905, 2220, ME 1088, 1158, 1391, 1435, RdeS

414, 700, SAY 2052, 2618, SS 1652, 3482, 3720.

Pithecellobium lanceolatum (Willd.) Benth.

CT 441, EM 32320, JR 718, ME 575, 2072, 2997.

Pithecellobium oblongum Benth.

ME 1173, 1348.

Pithecellobium seleri Harms

JR 2237, ME 1528, 1981, 2128.

Poeppigia procera C.Presl

JR 754, 2004, ME 741, 1035, 1266, 1417, SAY 2262, SS

$1854,2998,3474$.
Prosopis juliflora (Sw.) DC.

CH 1952, DS 5, MC 398, ME 1113, 1149, 1235, 1279.

Pterocarpus acapulcensis Rose

CH 1949, CP 20, JR 363, 551, 915, 1044, ME 3003, SS 2385 , 3338, 3463, 3568.

Pterocarpus rohrii Vahl

ET 1319, MC 360, ME 271, 2046, 2071, 2104, RGS 381, SAY 2094, SS 1258, 1655, 2006, 2039.

Ramirezella strobilophora (B.L.Rob.) Rose JFC 711, ME 1509, 2556, SS 1584, 2450.

Rhynchosia edulis Griseb.

NV 557, SAY 2587.

Rhynchosia minima (L.) DC.

CP 811, JFC 623, 671, 883, JR 2144, ME 3043, 3947, SAY 2747, SS 2579, 2615.

Rhynchosia phaseoloides (Sw.) DC. SS 2758.

Rhynchosia precatoria (Humb. et Bonpl. ex Willd.) DC. JR 2236, 2446, ME 5588, SS 3637, 3643.

Rhynchosia reticulata (Sw.) DC. CP 667, JP 116, SS 3601.

Senna alata (L.) Roxb. ME 4170, RdeS 709, SS 3722.

Senna atomaria (L.) H.S.Irwin et Barneby CH 1954, CT 442, 443bis, EM 32115, FL 2, JP 30, JR 377, ME 156, 1821, 1938, 2038, RdeS 733, SS 1791, 2886, 3398, 3476.

Senna cobanensis (Britton) H.S.Irwin et Barneby

DS 2, ME 205, 1246, 2493 bis, 3622.

Senna fruticosa (Mill.) H.S.Irwin et Barneby CP 181, 521, 666, FL 210, JFC 590, JP 37, JR 1629, 1632, 1911, 2531, ME 1102, 1499, 2106, 3118, NV 495, SAY 2614, SS 3034, 3467, 4174, YAC 161.

Senna hirsuta (L.) H.S.Irwin et Barneby JR 374.

Senna holwayana (Rose) H.S.Irwin et Barneby ME 1623, 3893, 3991, SAY 2654, SS 1619.

Senna mollissima (Humb. et Bonpl. ex Willd.) H.S.Irwin et

Barneby var. glabrata (Benth.) H.S.Irwin et Barneby ME 2111, RGS 470.

Senna nicaraguensis (Benth.) H.S.Irwin et Barneby FL 421, JFC 799, JP 100, 317, ME 1274, 1701, 1783, 2366, SS 3615, 3694.

Senna obtusifolia (L.) H.S.Irwin et Barneby CP 682, JFC 692, JP 69, ME 2635, 3552, 3649, 3865, SS $1498,3716$.

Senna occidentalis (L.) Link ME 102, 1090, 1206, 1467, NV 501, RdeS 729, SS 3723.

Senna pallida (J.Vahl) H.S.Irwin et Barneby var. geminiflora

H.S.Irwin et Barneby EM 32105.

Senna pallida (J.Vahl) H.S.Irwin et Barneby var. pallida ME 81, 1042.

Senna pendula (Willd.) H.S.Irwin et Barneby ME 112, 1448, 1638, 1660.

Senna reticulata (Willd.) H.S.Irwin et Barneby ME 111, 1100, 1198, 1256.

Senna serpens Greene NV 399, 449. 
Senna skinneri (Benth.) H.S.Irwin et Barneby

EM 32101, JR 774, 916, ME 420, 444, 3225, 3289, NV 462, SS 2434, 3140.

Senna uniflora (Mill.) H.S.Irwin et Barneby

CT 489bis, FL 366, 388, ME 3514, 4031, 4129, 5144, SAY

2485, SS 4025

Sesbania herbacea (Mill.) McVaugh

SS 4082, JR 1876, ME 938, 3690, NV 272.

Stizolobium pruriens (L.) Medik. var. pruriens

ME 2587.

Stizolobium pruriens (L.) Medik. var. utilis Wall. ex Wight

JR 474, ME 2609.

Stylosanthes guianensis (Aubl.) Sw.

JFC 935, 940.

Stylosanthes humilis Kunth

NV 404.

Stylosanthes viscosa (L.) Sw.

SS 3421.

Swartzia simplex (Sw.) Spreng. var. ochnacea (DC.) R.S.Cowan

JFC 1071, SS 3066.

₹ Tamarindus indica L.

DS 110.

Tephrosia cinerea (L.) Pers.

ME 4882, NV 274, 275, SAY 2438, SS 3207.

Tephrosia leiocarpa A.Gray

CP 670, 692, JFC 751, ME 914, 964, SS 4124.

Tephrosia macrantha A.Rob et Greenm. ex Pringle

ME 1394, 3781, 3805, 3926.

Tephrosia multifolia Rose

JP 211, ME 1593.

Tephrosia rhodantha Brandgee

SS 1545.

Tephrosia tenella A.Gray

ME 2393, 3625, 3985.

Tephrosia vicioides Schltdl.

JR 928, ME 668, 3804, 5391.

Teramnus labialis (L.f.) Spreng.

JFC 704, 1067.

Teramnus uncinatus (L.) Sw.

CP 711, 803, SS 3642.

Vigna adenantha (G.Mey.) Maréchal, Mascherpa et Stainer

JFC 905, ME 1084, 1185, 1234, 1330, SS 2500.

Vigna linearis (Kunth) Maréchal, Mascherpa et Stainer

JFC 948, JFC 954, SS 2493, 2528, 3088.

Vigna peduncularis (Kunth) Fawc. et Rendle

CP 721.

Vigna speciosa (Kunth) Verdc.

ME 1264.

Zapoteca alinae H.M.Hern.

NV 290.

Zapoteca formosa (Kunth) H.M.Hern.

ME 2391, 3593, 3700, 5230.

Zornia thymifolia Kunth

CP 649, 683.

Género nuevo

ME 238, 263, 456, 600, JR 2513, 2563, SAY 2056, 2202, 2203, 2204, SS 1773, 2922, 2962, 3308.

\section{LOASACEAE}

Gronovia scandens L.
CP 406, 416, JFC 872, ME 529, 824.

Mentzelia aspera L.

JR 901, ME 722, 2358.

LOGANIACEAE

Polypremum procumbens $\mathrm{L}$.

ME 3047.

Spigelia humboldtiana Cham. et Schltdl.

JFC 927.

LORANTHACEAE

Struthanthus interruptus (Kunth) G.Don

ME 2462, 2519.

LYTHRACEAE

Adenaria floribunda Kunth

JR 2480, ME 3166, SS 3021.

MALPIGHIACEAE

Bunchosia discolor Turcz.

ASA 42, 84, CP 36, 655, JR 764, 787, 1881, ME 406, 1000 , 2228, 2325, SS 1808, 2141, 2297.

Bunchosia lanceolata Turcz.

JFC 748, JR 882, ME 1145, 1433, 1863, 2359.

Bunchosia montana A.Juss.

JR 434.

Bunchosia palmeri S.Watson

SS 1555.

Byrsonima crassifolia (L.) Kunth

DS 58, JP 388, 438, ME 1795.

Callaeum malpighioides (Turcz.) D.M.Johnson

JR 2165.

Galphimia gracilis Bartl.

ME 650, 1349, 2389.

Gaudichaudia albida Schltdl. et Cham.

CP 75, 713, JR 1014, ME 904, 934.

Gaudichaudia aff. cycloptera (DC.) W.R.Anderson

JR 1204, ME 2073.

Heteropteris cotinifolia A.Juss.

JR 1081, ME 2545.

Heteropteris eglandulosa A.Juss.

ME 3115.

Heteropteris laurifolia (L.) A.Juss. JP 429, ME 332, SAY 2833, SS 2854.

Lasiocarpus sp.

JR 2231, ME 1074, 2759.

Malpighia glabra L.

EM 32355, JFC 634, ME 159, 1350, 1566, 1820, SS 1624, 2005.

Malpighia mexicana A.Juss.

ASA 51, ME 403, 539, SS 1560, 2059.

Malpighia ovata Rose

ME 2539.

Stigmaphyllon sp.

JFC 815, JR 1999, 2107, ME 941.

Tetrapterys heterophylla (Griseb.) W.R.Anderson

MC 352, ME 1765, 1774, SS 1638, 2663, 2679.

MALVACEAE

Abutilon aff. andrieuxii Hemsl.

JFC 880. 
Abutilon macvaughii Fryxell

SS 2001.

Anoda cristata (L.) Schltdl.

JFC 778.

Anoda lanceolata Hook. et Arn.

JFC 1077.

Anoda pedunculosa Hochr.

CP 656, JFC 541, 759, JR 1041, ME 3739.

Bastardiastrum gracile (Hochr.) D.M.Bates

JFC 814.

Gaya minutiflora Rose

FL 137.

Gossypium aridum (Rose et Standl. ) Skovst.

EM 32046, JFC 570, JR 557, 813, 2138, ME 1380, 1462,

1494, 2544, SS 1637, 1768, 2166.

Hampea mexicana Fryxell

FL 61.

Herissantia crispa (L.) Brizicky

JFC 567, ME 1117, 2626, SS 2637.

Hibiscus kochii Fryxell

JR 332, ME 673, SS 1606, 1995, 2031, 2557.

‡ Hibiscus rosa-sinensis $\mathrm{L}$.

JFC 715.

¥ Hibiscus sabdariffa L.

JFC 972.

Kosteletzkya depressa (L.) O.J.Blanch., Fryxell et D.M.Bates

ME 130, 723, 1850, 3050, RGS 383.

Malachra capitata (L.) L.

ME 99, 126, 803, 826.

Malachra fasciata Jacq.

JFC 638, 770.

Malvastrum americanum (L.) Torr.

ME 981, 1001.

Malvastrum coromandelianum (L.) Garcke

FL 151, JFC 622, ME 506, 2678.

Malvaviscus arboreus Cav. var. mexicanus Schltdl.

ME 967, 1202, 1252, 1320.

Pavonia paniculata Cav.

JFC 892, ME 2344.

Pavonia sidaefolia Kunth

FL 126, ME 968, 2733, 2807.

Sida acuta Burm.f.

CP 64, JFC 565, JR 867, 937, ME 622, 1915, 2300, 2349.

Sida ciliaris $\mathrm{L}$.

JFC 736, SS 1499, 1828.

Sida glabra Mill.

JFC 723, 729, SS 2564.

Sida jussieana DC.

JFC 621, 1072.

Sida linifolia Juss. ex Cav.

JFC 923.

Sida prolifica Fryxell et S.Koch

ME 788.

Sida rhombifolia L.

JFC 641, 649, 797, 907, JR 2526, ME 1013, 1204, 1319, 1591.

Sida urens $\mathrm{L}$.

JFC 656.

Wissadula amplissima (L.) R.E.Fr.

$$
\text { CP } 703 .
$$

MELASTOMATACEAE

Arthrostemma ciliatum Pav. ex D.Don

SAY 2500.

Clidemia hirta (L.) D.Don

EM 32307, SAY 2548.

Conostegia xalapensis (Bonpl.) D.Don ex DC. EM 32118, ME 1797, 1836, SAY 2295.

Miconia laevigata (L.) D.Don JR 2452.

Miconia schlechtendalii Cogn.

SAY 2387.

Miconia sylvatica (Schltdl.) Naudin

SAY 2522.

MELIACEAE

Cedrela salvadorensis Standl.

EM 32045, ET 1280, 1464, FL 62, JR 447, 2094, SS 1526 , 2226, 2231.

Guarea glabra Vahl

EM 32282, FL 27, 40, 83, JR 2156, SS 2793, 2822.

₹ Melia azedarach $\mathrm{L}$.

ME 10, 1886, 1940, 2640.

Swietenia humilis Zucc.

JR 350, 848, 879, ME 2018, SS 2066.

Trichilia americana (Sessé et Moc.) T.D.Penn.

JR 827, ME 609, SS 1860, 3398.

Trichilia havanensis Jacq.

EM 32288.

Trichilia hirta L.

ME 2378.

Trichilia pallida Sw.

ME 3254.

Trichilia trifolia L.

CH 1955, CP 407, JR 2109, ME 1177, 1211, 1453, 1562, SS 1556, 2477, 3544.

Trichilia aff. multifoliola C.DC.

JR 2179, ME 2179.

MENISPERMACEAE

Cissampelos pareira L. JFC 648, 2284.

Hyperbaena mexicana Miers JFC 875, JR 2542, ME 1537, 1724, 1757, 2251, SS 1955, 2657, 2811.

MOLLUGINACEAE

Mollugo verticillata $\mathrm{L}$.

DS 31, JR 808, ME 1671, 1864, 2302, 2339, SS 1829.

MONIMIACEAE

Siparuna andina (Tul.) A.DC.

MC 356.

MORACEAE

Brosimum alicastrum Sw. EM 32315.

Dorstenia contrajerva L. FL 239, 297, JR 2407.

Dorstenia drakena L.

JR 1586, 1759, 1795, ME 2187, 2371, 3242, 3318, SS 1874. 
Ficus calyculata Mill.

FL 464, JR 2170.

Ficus citrifolia Mill.

EM 32306.

Ficus cotinifolia Kunth

CH 1914, JR 593, 712, 1080, 1214, ME 1881, 2126, 3735 , 3874, RGS 467, SS 1642, 1898, 2046, 2485.

Ficus insipida Willd.

EM 32085, 32295, JR 469, 863, 1209, 1607, ME 1711, 1834, 2320, 2952, NV 368, SS 1863.

Ficus maxima Mill.

FL 7, 19.

Ficus obtusifolia Kunth

FL 356, SS 2882.

Ficus ovalis (Liebm.) Miq.

DS 63.

Ficus pertusa L.f.

DS 78, EM 32286, FL 380, JR 467, 2215, 2462, 2562, ME

1268, 1295, 1832, 2513, SS 1650.

Ficus petiolaris Kunth

JR 2498.

Ficus tecolutensis (Liebm.) Miq.

SS 2838.

Ficus trigonata L.

EM 32062, 32150, ME 2322, 2466, 2522, 3131, RdeS 704.

Ficus tuerckheimii Standl. SS 2732.

Maclura tinctoria (L.) D.Don ex Steud.

ASA 65, ET 1307, JR 444, 751, ME 2080, 2105, 2188, 2223 , SS 1622, 1783, 1802, 1883, YAC 165.

Trophis mexicana (Liebm.) Bureau

FL 69, SS 2724.

Trophis racemosa (L.) Urb.

ME 65, 953, SS 2786, 2844.

\section{MYRSINACEAE}

Ardisia revoluta Kunth

EM 32281, SS 1597.

\section{MYRTACEAE}

Eugenia aff. uniflora L.

SS 2806.

Myrcianthes fragrans (Sw.) McVaugh

DS 76.

Psidium guajava L.

SS 1251, 2857, 4437.

Psidium guineense $\mathrm{Sw}$.

ME 2276.

‡ Syzigium jambos (L.) Alston.

SS 2523, 2810.

\section{NYCTAGINACEAE}

Boerhavia coccinea Mill.

JFC 550, ME 649, 676, 1917, 2305, SS 1492, 2558.

Boerhavia diffusa L.

CP 628, ME 1879.

Boerhavia erecta L.

ASA 61, JFC 818, JR 806, 1600, 2382, ME 1702, 2306, 2363, 2624.
Commicarpus scandens (L.) Standl.

ME 1898, 5668, SS 2648,

Mirabilis laevis (Benth.) Curran

CP 673, JFC 1098, ME 2324, SS 2542.

Neea psychotrioides Donn.Sm.

ASA 83.

Neea stenophylla Standl. EM 32350.

Okenia hypogaea Schltdl. et Cham.

JFC 856, ME 419, 438, 2216, 2384, SS 1889.

Pisonia aculeata L. CP 198, ME 1478, 1563, 1762, 2059, RGS 462, SS 1957, 1987.

Pisonia capitata (S.Watson) Standl. ME 3543.

Pisonia aff. macranthocarpa (Donn.Sm.) Donn.Sm. ME 2328.

Salpianthus arenarius Humb. et Bonpl. CP 180, ME 120, 150, 163, 1880, SS 1608, 2014.

Salpianthus purpurascens (Cav. ex Lag.) Hook. et Arn. ME 50.

Torrubia macrocarpa Miranda CP 15, EM 32068, JR 426, 721, MC 377, ME 290, 308, 2180, 2274, SS 2027, 2426, YAC 143.

\section{NYMPHAEACEAE \\ Nymphaea pulchella DC. ME 530, 1742.}

\section{OCHNACEAE}

Ouratea acuminata (DC.) Engl. SS 2783, 2813.

\section{OLACACEAE}

Ximenia americana L. EM 32128, 32133, 32145, JR 337, 2251, ME 146, 2131, SS 1769.

\section{ONAGRACEAE}

Hauya elegans DC. subsp. barcenae (Hemsl.) P.H.Raven et Breedlove

JR 1627, ME 458, 484, 693, 2662.

Oenothera rosea L'Hér. ex Aiton

SS 2743.

OPILIACEAE

Agonandra obtusifolia Standl.

ME 1984, 2137, 2680, SS 2250.

Agonandra racemosa (DC.) Standl. CH 1926, ME 1838, 2735, 2790, SS 2843.

OXALIDACEAE

Oxalis yucatanensis (Rose) R.Knuth CP 686, EM 32362, JFC 610, 699, 725, 773, JR 807, 1806, ME 2304, 2732.

PAPAVERACEAE

Bocconia arborea S.Watson JP 294. 
PASSIFLORACEAE

Passiflora biflora Lam.

ME 1333, 2594, 2613, 2713.

Passiflora coriacea Juss.

JFC 597, 827, 1100, ME 73, 899, 1635, 2479, RGS 471, SS

1559, 1967, 1990, 2646.

Passiflora filipes Benth.

JFC 822, JR 2008, 2322, SS 1567.

Passiflora foetida L. var. gossypiifolia (Desv. ex Ham.) Mast.

ASA 49, JR 382bis, MC 394, ME 214, 1354, 1650, 2311.

Passiflora foetida L. var. hastata (Bertol.) Mast.

JR 382, 775, 830, SS 1632.

Passiflora foetida L. var. nicaraguensis (Killip ex Standl.) Killip CP 310, ME 1157, 1530, 1567, 1627, SS 1910.

Passiflora goniosperma Killip

JFC 882.

Passiflora holosericea L.

EM 32100.

Passiflora mexicana Juss.

ME 794.

Passiflora porphyretica Mast.

EM 32153.

\section{PEDALIACEAE}

Martynia annua L.

DS 16, JR 816, 1010, 1636, ME 508, 546, 2241, 2318.

PHYTOLACCACEAE

Agdestis clematidea DC.

JR 1032, ME 2182, 2240, 2252, 2333.

Petiveria alliacea L.

JR 1031, ME 576, 721, 3281, SS 1872.

Rivina humilis $\mathrm{L}$.

CP 50, DS 103, JR 759, ME 498, 1988, 1990, 3101, SS 1873.

Rivina aff. octandra L.

ME 2765.

Stegnosperma cubense A.Rich.

EM 32123, SS 2649.

\section{PLANTAGINACEAE}

Plantago major L.

ME 5990.

PLUMBAGINACEAE

Plumbago scandens L.

FL 35, SS 1781.

\section{PODOSTEMACEAE}

Marathrum schiedeanum (Cham.) Tul.

ME 1971.

\section{POLEMONIACEAE}

Cobaea biaurita Standl.

CP 693, JFC 612.

Loeselia ciliata L.

JFC 543, 1079, JP 237, 293, JR 1086, ME 1877, 3939, 4000, 4070.

Loeselia glandulosa (Cav.) G.Don

JP 292.
Loeselia mexicana (Lam.) Brand

DS 60.

\section{POLYGONACEAE}

Antigonon cinerascens M.Martens et Galeotti

JFC 868, ME 143, 171, 2084, 5902.

Antigonon flavescens S.Watson

JFC 733, ME 2603, 2705, 2821, 5872, SS 1507, 2556, 4428.

Coccoloba barbadensis Jacq.

EM 32079, 32285, SS 2634.

Coccoloba liebmannii Lindau

CP 306, FL 98, JFC 599, 737, 1093, JR 2104, ME 2453, 2518, 2568, 2689, SS 2571.

Coccoloba venosa L.

JFC 857.

Podopterus cordifolius Rose et Standl.

JFC 848, ME 155, 162, 170, 2687, SS 2011.

Podopterus mexicanus Bonpl.

ME 2976.

Ruprechtia fusca Fernald

CH 1919, JFC 765, JR 2259, ME 2507, SS 1276, 1592.

PORTULACACEAE

Portulaca oleracea L. ME 2902, SS 1978.

Talinum paniculatum (Jacq.) Gaertn. CP 61, ME 2259.

Talinum triangulare (Jacq.) Willd. CP 49, JR 458, ME 2260, SS 1812.

PUNICACEAE

₹ Punica granatum $\mathrm{L}$. DS 111.

RANUNCULACEAE

Clematis dioica $\mathrm{L}$. JFC 965, JR 2154.

RHAMNACEAE

Ceanothus coeruleus Lag.

FL 11.

Colubrina aff. triflora Brongn. ex Sweet JFC 571, ME 845, 871, 2376.

Gouania conzattii Greenm. ME 684, 991, SS 1510.

Gouania eurycarpa Standl. ME 726, 751, 785, 2398, SS 2476.

Gouania lupuloides (L.) Urb. CP 76, JFC 698, ME 642, SS 1843, 2136.

Gouania polygama (Jacq.) Urb. SS 3376.

Gouania aff. rosei Wiggins ME 891, 1037.

Karwinskia calderonii Standl. ME 2219, 2517.

Karwinskia humboldtiana (Willd. ex Roem. et Schlt.) Zucc. ET 1463, JR 729, 875, ME 585, 641, 1621, SS 1282.

Ziziphus amole (Sessé et Moc.) M.C.Johnst.

JR 417, 835, 858, ME 1564, 2169, 2386, 2474, SS 1278, 2474, YAC 126. 
Ziziphus obtusifolia (Hook. ex Torr. et A.Gray) A.Gray. var. canescens (Gray) M.C.Johnst.

JR 364.

\section{RUBIACEAE}

Alibertia edulis (Rich.) A.Rich. ex DC.

JFC 946, JP 17, JR 1731, ME 1790, NV 297, 513, SAY 2355, SS 3093, 3368, 3918, 4132.

Borreria laevis (Lam.) Griseb.

ME 5117.

Borreria vegeta Standl. et Steyerm.

CP 722.

Borreria verticillata (L.) G.Mey.

JR 949, ME 3681.

Bouvardia sp.

SAY 2098.

Calycophyllum candidissimum (Vahl) DC.

CP 167, ET 1305, FL 60, JFC 876, JR 1045, ME 1299, 1416, 1572, SS 1595.

Chiococca alba (L.) Hitchc.

CT 459b, JFC 674, JR 1998, ME 2512, SS 4000.

Chomelia protracta (Bartl. ex DC.) Standl.

EM 32308.

Chomelia spinosa Jacq.

EM 32147, 32284, JR 470, ME 2270, RGS 464.

Crusea calocephala DC.

NV 301, SS 2517.

Crusea coccinea DC. var. breviloba Loes.

FL 444.

Crusea coccinea DC. var. coccinea

FL 36.

Crusea hispida (Mill.) B.L.Rob.

JP 14, NV 372.

Crusea lucida Benth.

SAY 2661.

Crusea parviflora Hook. et Arn.

CP 206, ME 4090.

Crusea wrightii var. angustifolia W.R.Anderson

NV 414.

Deppea sp.

SS 3300.

Diodia apiculata (Willd. ex Roem. et Schult.) K.Schum.

ME 3302, SS 4029.

Diodia teres Walter

CP 469, NV 451, SS 3105.

Exostema caribaeum (Jacq.) Roem. et Schult.

ME 2329, SS 2149, 2359.

Exostema mexicanum A.Gray

SAY 2209.

Galium aschenbornii Nees et S.Schauer

FL 125, 152.

Genipa americana L.

ET 1287, JR 592, 1750, 2515, NV 295, SS 3084, 3112, 4269.

Gonzalagunia panamensis (Cav.) K.Schum.

FL 206, 275, SAY 2547, SS 3076, 3641, 4143.

Guettarda elliptica Sw.

ASA 86, JR 753, ME 486, 678, SS 2032, 2055, 2333.

Hamelia patens Jacq. var. glabra Oerst.

JR 840.
Hamelia patens Jacq. var. patens

CP 733, JR 2326, SS 2788.

Hamelia versicolor A.Gray

DS 96, JR 943, ME 2181, 2229, 2247, 2332, SS 1504, 1850, 1890.

Hintonia latiflora (Sessé et Moc. ex DC.) Bullock

EM 32047, JR 462, 479, 722, 887, ME 322, 347, 1109, 1458, SS 1312, 1983, 2024, 2355, 2373, YAC 169.

Hoffmannia sp.

FL 123.

Mitracarpus hirtus (L.) DC.

CP 92, JFC 909, JP 118, ME 892, 3634, SS 3401, 4127.

‡ Ophiorrhiza mitreola L.

SS 3522.

Pentodon pentandrus (Schumach. et Thonn.) Vatke

ME 1867.

Psychotria erythrocarpa Schltdl.

EM 32289, ME 4734, SS 3129.

Psychotria horizontalis Sw.

ET 1274, FL 185, SAY 2337, SS 4183.

Psychotria mexiae Standl.

ASA 81, FL 114.

Psychotria microdon (DC.) Urb. ME 621, 3299.

Psychotria nervosa Sw. SS 3019.

Psychotria pubescens Sw. FL 226, SAY 2523.

Psychotria quinqueradiata Pol. NV 509, SAY 2177.

Psychotria tenuifolia Sw. JR 2423, ME 4925, SS 2790.

Randia armata (Sw.) DC. JR 1782.

Randia laevigata Standl. CP 95, JR 782, ME 601.

Randia malacocarpa Standl. CP 637, CT 457a, JR 338.

Randia nelsonii Greenm. CP 651, JFC 1097, ME 153, 2165, 2530, SS 2058, 2147 , 2348, 2372.

Randia tetracantha (Cav.) DC. EM 32409, 32665, JR 847, 886, 960, ME 317, 395, 615, 2571, SAY 2811, SS 2441, YAC 141.

Randia thurberi S.Watson JR 766, ME 366, 414, 560, SS 1895.

Richardia scabra L. EM 32188, 32456, 32478, JFC 628, 920, ME 1920, 1996, 2282, 3547, NV 353, SAY 2582, SS 600.

Rondeletia leucophylla Kunth CP 200, 316, ET 1825, JFC 631, JR 351, ME 1536, 1696, 1969, RdeS 717.

Simira rhodoclada (Standl.) Steyerm. CP 94, EM 32055, JR 1043, ME 919, SS 1849, YAC 156.

Sommera grandis (Bartl. ex DC.) Standl. EM 32297.

Spermacoce assurgens Ruiz et Pav. CP 40, ME 3089, 4146, 4453, NV 327, SAY 1986, SS 3069. Spermacoce latifolia Aubl.

JFC 930. 
Spermacoce tenuior $\mathrm{L}$.

ME 517, 1737, 2580, 5170.

\section{RUTACEAE}

‡ Citrus sinensis (L.) Osbeck

DS 105.

Esenbeckia berlandieri Baill. ex Hemsl. subsp. acapulcensis (Rose) Kaastra

CP 14, JR 1830, ME 1059, 2440.

Esenbeckia berlandieri Baill. ex Hemsl. subsp. litoralis (Donn.Sm.) Kaastra

CT 411, 428, 499, ASA 43, CP 653, CT 467b, EM 32359, JFC 824, 847, JR 1823, 1824, 1825, 1892, ME 540, 2225, 2292, 2400, SS 1568, 1893, 2314, 2635.

Esenbeckia collina Brandegee CP 25, JR 1211, 410, 888, ME 324, 369, 639.

\section{SALICACEAE}

Salix humboldtiana Willd.

DS 84, ME 1818, 1857, 1991, 2504.

Salix taxifolia Kunth

EM 32087.

\section{SAPINDACEAE}

Cardiospermum halicacabum L. ASA 89, CT 439, EM 32649, JFC 718, ME 1495, 1697, 2704, 2994, NV 493, SAY 2423, 2783, SS 1547, 2621, 2951, 3536.

Cupania dentata DC.

FL 179, ME 2069.

Cupania glabra Sw.

JR 1794, SAY 1967, 2066, 2489.

Cupania aff. mollis Standl.

SS 2722.

Paullinia costaricensis Radlk.

ME 2830.

Paullinia cururu L.

FL 379, JR 1594, 1781, ME 2286, 3240, SAY 2254, 2466,

2484, SS 2308, 3227, YAC 154.

Paullinia fuscescens Kunth

JP 157, ME 181, 2056, 2679, 4443, RGS 402, SS 2048, 4424.

Paullinia tomentosa Jacq.

MC 353, SAY 1964, 2078, 2079.

Sapindus saponaria L.

CH 1927, FL 167, JR 602, ME 1810, 1884, 2043, 5011.

Serjania atrolineata C.Wright

JR 2181, ME 2703.

Serjania cardiospermoides Schltdl. et Cham. CP 484, SS 2457.

Serjania aff. goniocarpa Radlk.

SS 1529.

Serjania lobulata Standl. et Steyerm.

JR 898, 2222, ME 887, 1107, 2280,.

Serjania paniculata Kunth

CP 194

Serjania racemosa Schumach.

CP 97, ME 917.

Serjania triquetra Radlk.

FL 386.

Serjania yucatanensis Standl.

JR 2481.
Talisia oliviformis (Kunth) Radlk.

JR 2201.

Thouinidium decandrum (Bonpl.) Radlk.

JR 2096, 2211, 2229, ME 2607, 5575, 5667, 5899, RGS 388, SS 1252, 2660.

Urvillea ulmacea Kunth ME 782, SS 2594.

SAPOTACEAE

Bumelia celastrina Kunth CH 1960, JR 1092, 2105, ME 2510, 5928.

Manilkara zapota (L.) P.Royen ME 57, 1769, 2682.

₹ Pouteria sapota (Jacq.) H.E.Moore et Stearn. SS 2699

Sideroxylon capiri (A.DC.) Pittier CH 1943, EM 32070, JR 2217, 2325, 2341, ME 468.

Sideroxylon stenospermum (Standl.) T.D.Penn. EM 32144, 32155, 32391.

SAXIFRAGACEAE

Heuchera orizabensis Hemsl. FL 154.

SCROPHULARIACEAE

Bacopa monnieri (L.) Wettst. ME 1159, 2064.

Capraria biflora L. ME 502, 1590, 1739.

Capraria saxifragaefolia Schltdl. et Cham. SS 1521.

Mecardonia procumbens (Mill.) Small JFC 767, SS 2850.

Penstemon gentianoides (Kunth) Poir. FL 103.

Russelia coccinea (L.) Wettst. JR 2241, ME 1689, 1843.

Russelia sarmentosa Jacq. ME 2734

Russelia standleyi Carlson JR 2225, ME 1500, RdeS 730.

Schistophragma intermedia (Gray) Pennell ME 3076.

Scoparia dulcis $\mathrm{L}$.

JFC 605, ME 682, 779, 2797, 5653, SS 2593, 2651.

Stemodia durantifolia (L.) Sw. ME 118, 1603.

SIMAROUBACEAE

Alvaradoa amorphoides Liebm. JP 278, JR 559, 1054, ME 942, 1280, 1571,

Picramnia xalapensis Planch. FL 431, JR 1769.

Recchia mexicana Moc. et Sessé ex DC. ET 1282, JR 2088, ME 272, 1379, 1406, 1560, SS 1288, 1295, $1612,2678$.

SOLANACEAE

Capsicum annuum L. var. glabriusculum (Dunal) Heiser et Pickersgill ASA 90, JR 371 ME, 3132, SS, 1851. 
Cestrum nocturnum L.

JR 1774, 2395, ME 4920, SS 2737, 2792, 3656, 3686.

Datura discolor Bernh.

JR 369.

Lycianthes lenta (Cav.) Bitter

JR 1919, 2495, ME 134, 1095, 1237, 1343, SS 3691.

Lycopersicon esculentum Mill. var. leptophyllum (Dunal)

D'Arcy

ME 1304, 1835, 2711, 4145.

Solanum aculeatum St.-Lag.

CP 449, EM 32499.

Solanum aculeolatum M.Martens et Galeotti

ME 737, 3197.

Solanum adscendens Sendtn.

ASA 64, JR 1591, ME, 700, 3117, SS 2981.

Solanum americanum Mill.

FL 160, JR 2164, 2412, ME 1444, 1732, 1908, 1995, SAY

2503, SS 3970.

Solanum aturense Dunal

SAY 2610.

Solanum campechiense L.

ME 1853, 4034, 4254, 4275.

Solanum candidum Lindl.

ME 4084.

Solanum citrinum M.Nee

JR 2536, ME 1899.

Solanum dulcamaroides Dunal

FL 340, JP 16, SS 4001, 4158.

Solanum erianthum D.Don

ME 1091, 1140, 1473, 1498.

Solanum esculentum Dunal var. aculeatum Dunal

ME 2686.

Solanum ferrugineum Jacq.

FL 145.

Solanum gardneri Sendtn.

ASA 56, CP 30, MC 393, ME 1209, 1897, 2526, 3381, SAY 2658.

Solanum glaucescens Zucc.

JR 1818, ME 301, 319, 1434, 3155, SS 1896, 2321, 4073.

Solanum seaforthianum Andrews

JR 2525.

Solanum tampicense Dunal

ME 3029, 4253, 4595.

Solanum torvum Sw.

ME 620, 1436, 3450, 4395, SS 2727, 3744.

Solanum umbellatum Mill.

JFC 1070, ME 977, SS 3679.

Solanum wrightii Benth.

FL 34.

\section{STERCULIACEAE}

Ayenia palmeri S.Watson

CP 2, JR 786, ME 739, SS 1886, 2369.

Ayenia pusilla L.

CP 402, 468, EM 32552, JR 1596, 1734.

Byttneria aculeata (Jacq.) Jacq.

CP 422, 487, 657, JR 375, 793, 1890, ME 1946, 2330, 2367, 3390, NV 336, SS 894, 1532, 1881, 2963.

Byttneria catalpifolia Jacq.

SS 4145.
Guazuma ulmifolia Lam.

ASA 52, CT 415, 447, 473, EM 32076, FL 136, 411, JFC 589, JP 402, 424, JR 361, 1016, 1755, 2332, ME 1104, 1156, 1178, 1249, RdeS 701, RGS 406, SS 2018.

Helicteres baruensis Jacq. ME 2091, SS 4433.

Helicteres mexicana Kunth EM 32098, JP 77, ME 1798, 2343, 2824,

Melochia nodiflora Sw.

CP 81, 182, 819, CT 484, ME 91, 1044, 1086, SS 2667.

Melochia pyramidata L.

EM 32213, ME 1244, 1389, 1748, 1997.

Melochia tomentosa L.

EM32140, JR 383, 459, ME 149, 1179, 2476, 3703, SS 1968, 3577.

Waltheria indica $\mathrm{L}$.

CP 80, CT 467a, ME 1599, 2542, 2636, RGS 385.

Waltheria preslii Walp.

EM 32167, JFC 849, ME 1222, 1534, 1550, 1985.

THEOPHRASTACEAE

Jacquinia donnell-smithii Mez

JR 360, SS 1250.

Jacquinia macrocarpa Cav. CH 1918, CP 168, EM 32044, JR 2136, ME 266, 2103, 2121.

TILIACEAE

Corchorus aestuans L.

ME 3550, ME 651, 836,

Corchorus hirtus L.

ME 3595, 3682.

Corchorus siliquosus L.

ME 982, 4591.

Corchorus trilocularis L. ME 3604, 3658.

Dicraspidia sp.

ET 1291.

Heliocarpus donnellsmithii Rose CP 19, JFC 722, JR 1051, ME 86, 1181, 1407, 2543.

Heliocarpus mexicanus (Turcz.) Sprague CT 479bis, ME 2778, SS 1272, 1530, 1587, 1643.

Heliocarpus occidentalis Rose CP 663, JFC 553.

Heliocarpus cf. palmeri S.Watson ME 3648.

Luehea candida (Moc. et Seseé ex DC.) Mart. CP 186, ET 1272, JR 379, 436, 914, 1047, ME 70, 1513, SAY 2235, 2607, SS 1246, 1782, 1858.

Trichospermum mexicanum (DC.) Baill. JFC 913.

Triumfetta bogotensis DC.

JFC 538, ME 78.

Triumfetta semitriloba Jacq. CP 719, JFC 808, ME 2610.

TRIGONIACEAE

Trigonia rasa Standl. et Steyerm. JR 1723.

TURNERACEAE

Erblichia odorata Seem.

FL 52, 84, MC 354. 
Turnera diffusa Willd. ex Schult.

EM 32462, JR 1021, SS 1950.

Turnera ulmifolia L.

JR 777, 1029, ME 445, 1663, 1947, 3637.

\section{ULMACEAE}

Aphananthe monoica (Hemsl.) J.-F.Leroy

JR 1801, SS 2832.

Celtis caudata Planch.

JR 1589, ME 432.

Celtis iguanaea (Jacq.) Sarg.

CP 44, 199, CT 448a, EM 32156, JR 757, ME 1213, 2172,

2220, 2230, RGS 461.

Trema micrantha (L.) Blume

CP 702, JP 285, SS 2461, 3915.

\section{URTICACEAE}

Boehmeria caudata Sw.

SS 3660.

Discocnide mexicana (Liebm.) Chew

JR 2309.

\section{VALERIANACEAE}

Valeriana clematitis Kunth

FL 16.

\section{VERBENACEAE}

Avicennia germinans (L.) L.

ME 285, 2063, YAC 151.

Bouchea nelsonii Grenzeb.

ME 670, 703, 828, 2362, SS 1549.

Clerodendron ligustrinum (Jacq.) E.Br.

EM 32121.

Lantana camara L.

ASA 63, CP 4, CT 502, ET 1289, 1473, JFC 709, JR 770, 853, ME 1747, 1960, 2204, 2643, SS 1518.

Lippia mcvaughi Moldenke

ME 3848.

Petrea volubilis L.

EM 32346, JP 339, 353, JR 2153, SS 2802.

Phyla nodiflora (L.) Greene

JR 817, ME 110, 116, 2107, 2338.

Priva lappulacea (L.) Pers.

EM 32216, ME 1743, 1911, 1999, 2434.

Stachytarpheta frantzii Pol.

CP 706, DS 39, JR 758, 1611, 1612, 1810, ME 39, 1314,

1734, 1916, RdeS 708.

Vitex hemsleyi Briq.

ASA 69, EM 32086, 32129, JP 428, JR 330, ME 213,SS 2000.

Vitex mollis Kunth

CP 305, EM 32135, JR 353, 437, 1206, 1207, MC 367, ME

371, 591, 1719, 2031, RdeS 714, SS 1892, 1979.

\section{VIOLACEAE}

Hybanthus mexicanus Ging.

SS 2349, 2481, 2968.

\section{VISCACEAE}

Phoradendron quadrangulare (Kunth) Griseb.

EM 32292, ME 2461.
VITACEAE

Ampelopsis mexicana Rose EM 32074, JR 1213, 2161, ME 316, 1766, 1932, 2097, SS 2035.

Cissus sicyoides $\mathrm{L}$. EM 32180, 32202, JR 354, 473, 2110, 2204, ME 1153, 1205 , 1393, 1693, RdeS 719, SS 1953.

\section{ZYGOPHYLLACEAE}

Guaiacum coulteri A.Gray CT 436, DS 45, ET 1281, 1313, JFC 846, JR 549, 1038, 2359, ME 1136, 1176, 1561, 1772, NV 283, RGS 387, SAY 2932, 2933, SS 1248, 1270, 1777, 1797, YAC 138.

Kallstroemia brachystylis Vail ASA 93, CP 480, EM 32360, ME 418, 2237, 2303, SS 2917.

Kallstroemia maxima (L.) Hook. et Arn.

CT 481bis, EM 32187, FL 221, 354, JR 2502, ME 1012, 2897.

Tribulus cistoides L.

ME 3532.

\section{LILIOPSIDA}

AGAVACEAE

Agave angustifolia Haw. ME 1219, 1353.

Agave rhodacantha Trel. ME 1517, SS 1966, 2063, 2550.

Manfreda littoralis A.García-Mend., A. Castañeda et S.Franco EM 32447, 32524.

\section{ARACEAE}

Anthurium aff. rzedowskii Croat JP 347.

Anthurium aff. schlechtendalii Kunth JR 2402.

Philodendron hederaceum (Jacq.) Schott DS 83, ME 861, 1151.

Pistia stratiotes L. SS 4205.

Xanthosoma robustum Schott JFC 867, ME 510.

ARECACEAE

Acrocomia mexicana Karw. ex Mart. EM 32059.

Chamaedorea pochutlensis Liebm. FL 183, 57, JR 2505, SS 3374.

Cryosophila nana (Kunth) Blume ex Salomon EM 32060, SAY 2240.

Sabal mexicana Mart.

EM 32130, ME 1945, SS 1958.

BROMELIACEAE

Billbergia macrolepis L.B.Sm. EM 32054, JR 865, ME 2153.

Billbergia pallidiflora Liebm.

JFC 970.

Bromelia cf. alsodes H.St.John SS 2057.

Bromelia karatas L. EM 32097, FL 78, SAY 2169. 
Bromelia palmeri Mez

CT 433, ME 2319.

Bromelia cf. pinguin L.

JR 738, ME 2124.

Bromelia plumieri (E.Morren) L.B.Sm.

ME 2152.

Fosterella micrantha (Lindl.) L.B.Sm.

JR 2177, MC 355, SS 2696.

Hechtia sp.

MC 359.

Pitcairnia oaxacana L.B.Sm.

JR 1829, SS 3068.

Tillandsia caput-medusae E.Morren

JR 2307.

Tillandsia concolor L.B.Sm.

JR 878, SS 2049.

Tillandsia fasciculata Sw.

EM 32043, SS 2023.

Tillandsia pseudobaileyi C.S.Gardner

ME 1496, SS 1919.

COMMELINACEAE

Commelina diffusa Burm.f.

JFC 784, ME 1731, 1866, 2581, 2799, 2903, SS 2849.

Commelina erecta L.

CP 452, 456, 481,490, 674, EM 32470, 32473, JFC 342, 596, 727, 1073, 1606, 1610, JR 554, 749, 799, 891, 902, ME 437,

442, 1289, 1730, 2257, SS 1491, 1811, 1845, 2561, 2590.

Tinantia leiocalyx C.B.Clarke ex Donn.Sm.

CP 417, 778, EM 3247, JFC 792, ME 729.

Tinantia longipedunculata Standl. et Steyerm.

CP 502, EM 32493, ME 2254.

Tradescantia zanonia (L.) Sw.

CP 750, JR 1779.

\section{COSTACEAE}

Costus pictus D.Don

FL 229, JR 1588.

Costus scaber Ruiz et Pav.

FL 247.

\section{CYPERACEAE}

Cyperus canus J.Presl et C.Presl

ET 1826, JFC 583, 803, JR 456, 832, 2356, ME 88, 1474,

1515, 1678, 1692, 1953, RdeS 715, SS 2823.

Cyperus compressus L.

CP 485, EM 32467, 32477, JR 820, ME 3504.

Cyperus dentoniae G.C.Tucker

NV 356.

Cyperus esculentus L.

SS 3223.

Cyperus fugax Liebm.

JR 957, ME 818, SS 3222.

Cyperus hermaphroditus (Jacq.) Standl.

CP 432, 492, CT 498, JFC 554, 577, 604, JR 1882, ME 2011, 2336, SS 2597, 3011.

Cyperus humilis Kunth

JFC 667, ME 1207, 1606, 1676.

Cyperus laxus Lam.

CP 759, 763, 779, JFC 912, 953, JR 1808, ME 945, SS 3065.
Cyperus ligularis L.

NV 273, SS 2896.

Cyperus mutisii (Kunth) Andersson

SS 3083.

Cyperus ochraceus Vahl

FL 264, 349.

Cyperus odoratus L.

FL 240, 350, JFC 662, 788, ME 1016, SS 1514, 1907, 3489.

Cyperus oxylepis Ness

NV 270.

Cyperus rotundus $\mathrm{L}$.

EM 32204, ME 527,1326, 3465, 3617.

Cyperus surinamensis Rottb.

JFC 668, ME 3496.

Cyperus tenerrimus J.Presl et C.Presl

ASA 91, CP 511, EM 32459, JR 829, 958, 1615, 1933, ME

2262, NV 350, 490, SS 1833, 2899, 3524.

Cyperus tenuis Sw.

JFC 787, JR 917, ME 765.

Cyperus virens Michx. subsp. drummondii (Torr. et Hook.)

T.Koyama

ME 1082.

Eleocharis geniculata (L.) Roem. et Schult.

JR 2210, ME 840.

Eleocharis minutissima Britton

SS 3414.

Fimbristylis cymosa R.Br.

ME 1667, RdeS 718.

Fimbristylis dichotoma (L.) Vahl JFC 911, SS 3111.

Fimbristylis littoralis Gaudich. SS 3124.

Fuirena simplex Vahl

ME 1668.

Kyllinga odorata Vahl

FL 241.

Kyllinga pumila Michx. JR 1789.

Lipocarpha micrantha (Vahl) G.C.Tucker EM 32310.

Rhynchospora contracta (Nees) J.Raynal CP 56, JFC 640, JR 959, SS 3415, 3431.

Rhynchospora mexicana (Liebm.) Steud. NV 320.

Rhynchospora nervosa (Vahl) Boeck. NV 385, SS 3015.

Rhynchospora radicans (Schltdl. et Cham.) H.Pfeiff. subsp. microcephala (Bertero ex Spreng.) W.W.Thomas JFC 639.

Scleria lithosperma (L.) Sw.

JFC 933.

Scleria melaleuca Rchb. ex Schltdl. et Cham.

JFC 686, 944, JR 1807, NV 384, 550, SS 3359.

Scleria schiedeana Schltdl. NV 520, SS 3079.

DIOSCOREACEAE

Dioscorea insignis C.V.Morton et B.G.Schub. JP 162. 
Dioscorea mexicana Scheidw.

EM 32148, 32313, JP 3, ME 939.

Dioscorea nelsonii Uline ex R.Knuth CP 82, EM 32421, JR 1634, 1780, ME 2263.

Dioscorea polygonoides Humb. et Bonpl. ex Willd. JP 114.

Dioscorea remotiflora Kunth ME 946.

Dioscorea subtomentosa Miranda SS 2051.

\section{HELICONIACEAE}

Heliconia collinsiana Griggs SS 2733.

Heliconia latispatha Benth. JR 1760.

\section{IRIDACEAE}

Cipura paludosa Aubl. SS 3113.

Tigridia orthantha (Lem.) Ravenna FL 21.

\section{LILIACEAE}

Bomarea edulis (Tussac) Herb. JFC 926.

Hymenocallis littoralis (Jacq.) Salisb. ME 940.

\section{MARANTACEAE}

Calathea soconuscum Matuda

JR 1725.

Maranta arundinacea L.

CP 455, 715, JR 913,1608, 1906, SS 1540.

Thalia geniculata L.

JR 1219, ME 2000, 2492.

\section{ORCHIDACEAE}

Beloglottis costaricensis (Rchb.f) Schltr. SAY 1972.

Beloglottis cf. mexicana Garay et Hamer SS 2789.

Bletia lilacina A.Rich. et Galeotti SS 2871.

Bletia roezlii Rchb.f.

NV 379, SS 2504.

Brassavola cucullata (L.) R.Br. SAY 2264.

Brassia signata Rchb.f. SAY 2192.

Campylocentrum porrectum (Rchb.f.) Rolfe EM 32294.

Cattleya aurantiaca (Bateman ex Lindl.) P.N. Don SS 3663.

Chysis sp.

SAY 2909.

Clowesia dodsoniana Ag.León

ME 2154, SS 1765.

Encyclia cf. adenocarpon (La Llave et Lex.) Schltr. EM 32316.
Encyclia sp. nov. SS 4414.

Epidendrum cnemidophorum Lindl. SS 3654.

Epidendrum raniferum Lindl. JR 2387.

Habenaria cf. odontopetala Rchb.f. SAY 2596.

Habenaria pringlei B.L.Rob. FL 278.

Laelia rubescens Lindl. ME 3966, SS 1920.

Leochilus crocodiliceps (Rchb.f.) Kraenzl. SAY 1988.

Lockhartia sp. SAY 2191.

Maxillaria variabilis Bateman ex Lindl. JR 2427, SS 3662.

Myrmecophila galeottiana (A.Rich.) Rolfe ME 1540, SS 1921.

Notylia orbicularis A.Rich et Galeotti SS 2809.

Oncidium cebolleta (Jacq.) Sw. SS 1917.

Oncidium leleui R.Jiménez et Soto Arenas SS 2700, 3664.

Ornithocephalus cf. inflexus Lindl. JR 2426.

Pleurothallis ghiesbreghtiana A.Rich. et Galeotti SS 2705.

Polystachya foliosa (Lindl.) Rchb.f. SAY 2516.

Prosthechea cochleata (L.) W.E.Higgins JR 2388.

Prosthechea trulla (Rchb.f.) W.E.Higgins FL 56, JR 2506, SAY 2154, SS 2702.

Sacoila lanceolata (Aubl.) Garay EM 32300, SS 2872.

Trichocentrum ascendens (Lindl.) M.W.Chase et N.H.Williams SAY 2775.

Trichocentrum undulatum (Sw.) Ackerman et M.W.Chase SAY 2463.

POACEAE

Andropogon fastigiatus Sw.

JFC 910.

Anthephora hermaphrodita (L.) Kuntze CP 57, 466, 685.

Aristida adscensionis L. CP 409.

Aristida ternipes Cav. JFC 839.

Arundinella deppeana Nees ex Steud. JFC 945, 950.

Arundo donax $\mathrm{L}$. ME 629, JR 892.

Axonopus compressus (Sw.) P.Beauv. JFC 660, 899.

Bouteloua curtipendula (Michx.) Torr. ME 647, JFC 833bis. 
Bromus sp.

FL 10.

Cenchrus brownii Roem. et Schult.

JFC 647.

₹ Cenchrus ciliaris L.

ME 1646.

Cenchrus echinatus L.

ME 509.

Chloris barbata Sw.

CP 436.

Chusquea liebmannii E.Fourn.

JR 1727.

₹ Dactyloctenium aegyptium (L.) Willd.

CP 53, ME 494, 3544, SS 2583.

Digitaria bicornis (Lam.) Roem. et Schult.

CP 63, 509, EM 32210, JFC 586, 828, JR 1742, NV 342, SS 2592, 2598.

Digitaria ciliaris (Retz.) Koeler

JFC 637.

Digitaria insularis (L.) Fedde

EM 32217, JR 1783.

\# Echinochloa colona (L.) Link

CP 453, 462, ME 820, 1672, SAY 2449, SS 2584.

\# Eleusine indica (L.) Gaertn.

ME 628, 1679, NV 355, SS 3318.

Eragrostis amabilis (L.) Wight et Arn. ex Nees

ME 2595.

Eragrostis ciliaris (L.) R.Br.

CP 175, 644, 684, JFC 569, 578, ME 1669, 3642, NV 456

SS, 2614, 3317.

Eragrostis glomerata (Walter) L.H.Dewey

JFC 783, SS 3490.

Eragrostis maypurensis (Kunth) Steud.

JFC 898.

Gouinia virgata (J. Presl) Scribn.

JFC 591.

Hymenachne amplexicaulis (Rudge) Nees

ME 2491.

Ichnanthus pallens (Sw.) Munro ex Benth.

JFC 897.

Jouvea pilosa (J. Presl) Scribn.

JFC 853, NV 289.

Lasiacis divaricata (L.) Hitchc. var. leptostachya (Hitchc.)

Davidse

JFC 925.

Lasiacis oaxacensis (Steud.) Hitchc.

JFC 964.

Lasiacis procerrima (Hack.) Hitchc.

CP 720, 777, JFC 951, NV 551.

Lasiacis rhizophora (E.Fourn.) Hitchc.

SAY 2490.

Lasiacis ruscifolia (Kunth) Hitchc.

CP 426, 532, 533, JFC 665, 841, JR 1017, 1028, ME 993, 1300, 3698, NV 482.

Leptochloa panicea (Retz.) Ohwi subsp. brachiata (Steud.)

N.Snow

ME 1675.

Leptochloa virgata (L.) P.Beauv.

JFC 653.
Lithachne pauciflora (Sw.) P.Beauv.

JR 2398.

₹ Melinis minutiflora P.Beauv.

JFC 886.

₹ Melinis repens (Willd.) Zizka

CP 39, ME 534, NV 318.

Muhlenbergia sp.

JR 396.

Olyra latifolia L.

CP 717, JFC 655, SAY 2263, 2380, SS 3370.

Oplismenus burmannii (Retz.) P.Beauv. var. nudicaulis (Vasey)

McVaugh

CP 51, 636, 690, JFC 585, 600 JR 1035, SS 1908, 3485, 3521.

Oplismenus compositus (L.) P.Beauv.

JFC 693.

Panicum fasciculatum Sw.

CP 405, 458, 461, ME 1750, 2910, 3087, 3309, 3475, 3719, 3747, SS 3010.

Panicum hirticaule J.Presl

ME 495.

Panicum laxum Sw.

ME 1014, 1316, 3503.

Panicum maximum Jacq.

EM 32182, 32198, FL 329, ME 1749, 2486, SAY 2457.

Panicum muticum Forssk.

ME 2627.

Panicum pilosum Sw.

JFC 900, 1068, JR 1809, SAY 2332, 2381.

Panicum polygonatum Schrad.

ME 4923.

Panicum trichoides Sw.

CP 54, 457, 623, EM 32199, JFC 575, 588, 603, 632, 902, 1069, JR 908, 951, ME 771, 3471, 3574, 3746, NV 458, SS 3486.

Paspalum botteri (E.Fourn.) Chase

JFC 658, SS 3009.

Paspalum clavuliferum C.Wright SS 4263.

Paspalum conjugatum P.J.Bergius SAY 2272, 2499.

Paspalum notatum Flüggé SAY 2285.

Paspalum plicatulum Michx. FL 259, SAY 2369, 2388, SS 3114.

Paspalum vaginatum Sw. ME 1166.

Pennisetum nervosum (Nees) Trin. SS 4278.

Pennisetum setosum (Sw.) Rich. CP 720 bis, JFC 963.

Schizachyrium sanguineum (Retz.) Alston JFC 916, 929.

Setaria geniculata P.Beauv. SAY 2373.

Setaria liebmannii E.Fourn. CP 48, 510, EM 32451, JR 803, ME 449, 2315, 3469, SS 312.

Setaria parviflora (Poir.) Kerguélen CP 726, JR 1790, ME 815, 1324, 4825, SS 3078. 
Silvia H. Salas-Morales, Alfredo Saynes-Vásquez y Leo Schibli

Setaria sphacelata (Schumach.) M.B.Moss ex Stapf et C.E.Hubb. SAY 2259.

Setaria tenax (Rich.) Desv. var. tenax JR 1729.

Tripsacum latifolium Hitchc. JP 129.

\section{PONTEDERIACEAE}

Eichhornia crassipes (Mart.) Solms ME 1688, 2067.

Pontederia sagittata C.Pres|

JR 1772. 